\title{
Exact hybrid-kinetic equilibria for magnetized plasmas with shearing flows
}

\author{
G. Guzzi ${ }^{1}$, A. Settino ${ }^{1,2}$, F. Valentini ${ }^{1}$, and F. Malara ${ }^{1}$ \\ ${ }^{1}$ Dipartimento di Fisica, Università della Calabria, ponte P. Bucci, cubo 31C, 87036 Rende (CS), Italy \\ e-mail: adriana.settino@unical.it \\ 2 Swedish Institute of Space Physics, Box 537, 75121 Uppsala, Sweden
}

Received 12 October 2020 / Accepted 20 November 2020

\begin{abstract}
Context. Magnetized plasmas characterized by shearing flows are present in many natural contexts, such as the Earth's magnetopause and the solar wind. The collisionless nature of involved plasmas requires a kinetic description. When the width of the shear layer is on the order of ion scales, the hybrid Vlasov-Maxwell approach can be adopted for this purpose.

Aims. The aim of this work is to derive explicit forms for stationary configurations of magnetized plasmas with planar shearing flows within the hybrid Vlasov-Maxwell description. Two configurations are considered: the first with a uniform magnetic field obliquely directed with respect to the bulk velocity and the second with a uniform-magnitude variable-direction magnetic field.

Methods. We obtained stationary ion distribution functions by combining single-particle constant of motions, which are derived through the study of particle dynamics. Preliminary information about the form of the distribution functions were analytically derived in considering a local approximation for the background electromagnetic field. Then a numerical method was set up to obtain a solution for general profiles.

Results. We determined explicit distribution functions that allow us to obtain profiles of density, bulk velocity, temperature, and heat flux. Anisotropy and agyrotropy in the distribution function were also evaluated. The stationarity of the solution during numerical simulations was checked in the uniform oblique magnetic field case.

Conclusions. The configurations considered here can be used as models for the Earth's magnetopause in simulations of the KelvinHelmholtz instability.
\end{abstract}

Key words. plasmas - magnetic fields - turbulence - instabilities - waves - interplanetary medium

\section{Introduction}

Shearing flows in a fluid or in a plasma are characterized by a velocity field, $\boldsymbol{u}$, which varies in a direction nearly perpendicular to $\boldsymbol{u}$. This kind of configuration is found in various astrophysical contexts. For instance, it is present in the interaction region between fast and slow streams of the solar wind (Bruno \& Carbone 2013), in the interface between the solar wind and a planetary (Sckopke et al. 1981; Fujimoto et al. 1998; Hasegawa et al. 2004) or a cometary magnetosphere (Ershkovich \& Mendis 1983; McComas et al. 1987; Malara et al. 1989), in the solar corona at the surface limiting coronal mass ejections (Foullon et al. 2011), and in the interface between astrophysical jets and the surrounding medium (Hamlin \& Newman 2013). In a magnetized plasma, a configuration with a shearing flow is unstable if the velocity jump, $\Delta u$, across the shear layer is larger than a certain threshold, which is on the order of the Alfvén velocity associated with the magnetic field component $B_{\|}$parallel to $\boldsymbol{u}$. In this case, a Kelvin-Helmholtz instability (KHI) develops (e.g., Chandrasekhar 1961). The KHI has been extensively studied at the Earth's magnetopause, where the solar wind plasma slips over a more static magnetosphere (Kivelson \& Chen 1995; Seon et al. 1995; Fairfield et al. 2000, 2003; Hasegawa et al. 2004, 2006; Nykyri et al. 2006). There, the parallel magnetic field is small enough to allow for the development of the instability. In the Earth's magnetosphere KHI determines several phenomena; for instance, it can favor the solar-wind plasma entry into the magnetosphere through magnetic reconnection that takes place in certain magnetic configurations (Eriksson et al. 2016; Nakamura et al. 2017; Sisti et al. 2019).

From a theoretical point of view, the development of KHI in a plasma has been largely studied, both in the framework of magnetohydrodynamics (MHD; e.g., Axford 1960; Walker 1981; Miura 1982; Contin et al. 2003; Matsumoto \& Hoshino 2004; Nakamura et al. 2004; Faganello et al. 2008) or by using a kinetic description (Pritchett \& Coroniti 1984; Matsumoto \& Hoshino 2006; Cowee 2009; Matsumoto \& Seki 2010; Nakamura et al. 2010, 2011, 2013; Henri et al. 2013; Karimabadi et al. 2013); for a review see also Faganello \& Califano (2017). A kinetic approach is more appropriate than MHD in the case of the Earth's magnetopause, where the scale length associated with the shear layer is of the order of ion scales.

Stable shearing flows are relevant within the problem of wave propagation in nonuniform plasmas. When a perturbation propagates in an inhomogeneous velocity or magnetic field, fluctuations at small scales are generated, leading to a fast wave dissipation. Moreover, couplings among different wave modes can take place. These effects have been widely studied both analytically and numerically using an MHD description (e.g, Mok \& Einaudi 1985; Lee \& Roberts 1986; Hollweg 1987; Califano et al. 1990, 1992; Malara et al. 1992, 1996a; Kaghashvili 1999; Tsiklauri et al. 2002; Landi et al. 2005; Kaghashvili et al. 2006; Kaghashvili 2007; Pucci et al. 2014), 
whereas, in the framework of hybrid simulations, the study of propagation and interaction of Alfvénic wave packets in an inhomogeneous medium have demonstrated the importance of both collisions and kinetic effects, which lead to the generation of anisotropies and agyrotropies in the distribution function (e.g., Pezzi et al. 2017a,b and references therein). Such mechanisms of small-scale production have been invoked in wave-based models of coronal heating (e.g., Petkaki et al. 1998; Malara et al. 2000, 2003, 2005, 2007), as well as to describe turbulence evolution at the interface between fast and slow solar wind (Roberts et al. 1992) or around the heliospheric current sheet (Malara et al. 1996b). More recently, the propagation of Alfvén waves on inhomogeneities at scales comparable with ion scales has been studied using both Hall-MHD and kinetic simulations (Vàsconez et al. 2015; Pucci et al. 2016; Valentini et al. 2017), including a case where the wave propagates in a shearing flow (Maiorano et al. 2020). It has been shown that the wave evolution leads to formation of kinetic Alfvén waves, which are believed to play a role in energy dissipation for collisionless plasmas, as well as in the generation of field-aligned ion beams (Valentini et al. 2017; Maiorano et al. 2020) that are similar to those observed in the solar wind (Marsch 2006).

Prior to studying either KHI or the wave propagation in a shear layer, there is the problem of building up a stationary configuration representing the shearing flow. This is a straightforward exercise to carry out within a fluid theory. On the other hand, if the plasma is noncollisional and the shear width is comparable with ion scales - as, for instance, at the magnetopause - a kinetic approach is more suitable than a fluid one. In that case, building a stationary velocity-sheared configuration represents a much more complex task. In the fully kinetic case, particle distribution functions (DFs) corresponding to shearing flows has been found for a magnetic field parallel to the bulk velocity (Roytershteyn \& Daughton 2008), a uniform perpendicular magnetic field (Ganguli et al. 1988; Nishikawa et al. 1988; Cai et al. 1990), and a nonuniform magnetic field (Mahajan \& Hazeltine 2000). Fully kinetic stationary solutions describing both force-free Harris sheets and non-force-free magnetic configurations have also been found (Allanson et al. 2016 and references therein). In spite of the existence of such exact stationary solutions, in many kinetic studies of the KHI a shifted Maxwellian (SM) has been used as unperturbed particle DF. However, since an SM is not an exact stationary state, this generates spurious oscillations that affect the development of the instability. In particular, Settino et al. (2020) recently showed that both linear and nonlinear stages of KHI are different when an exact stationary solution is employed instead of an SM to represent the same shearing flow. Using an exact stationary background is essentially also in the problem of wave propagation in shearing flows (Maiorano et al. 2020), otherwise spurious oscillations would superpose over waves, making it difficult to follow the wave evolution.

In the present paper, we deal with the problem of building up a stationary state representing a collisionless magnetized plasma with a shearing flow. We are interested in a situation where the length scale of the shear layer is of the order of ion scales (ion inertial length or Larmor radius). For this reason, we situate our study within the framework of the hybrid Vlasov-Maxwell (HVM) theory. In the HVM model, ions are kinetically represented while electrons are treated as a massless fluid (Valentini et al. 2007). This kind of representation has been largely employed to describe phenomena at scales larger than (or on the order of) ion scales (e.g., Cerri et al. 2016, 2017; Franci et al. 2015; Matthaeus et al. 2014; Pezzi et al. 2018;
Servidio et al. 2012, 2015; Valentini et al. 2011, 2016, 2017). In particular, Cerri et al. (2013) derived approximately stationary ion distribution functions based on the evaluation of finite Larmor radius effects in the ion pressure tensor; their method has been employed to describe temperature anisotropy in the presence of shearing flows (Cerri et al. 2014; Del Sarto et al. 2016). In a previous paper (Malara et al. 2018, hereafter, Paper I), within the HVM framework, we built up exact stationary configurations characterized by a shearing flow with a uniform magnetic field that is either parallel or perpendicular to the plasma bulk velocity. The method is based on the determination of a stationary ion DF in terms of single-particle constants of motions. Such solutions have been employed to study the evolution of an Alfvénic disturbance in a shearing flow in the case of a parallel magnetic field (Maiorano et al. 2020), as well as the development of KHI (Settino et al. 2020) in the case of a perpendicular magnetic field.

Here, we extend the stationary solutions found in Paper I to more general magnetized shearing flow configurations. Namely, a shearing flow with a uniform obliquely directed magnetic field or variable-directed uniform-intensity magnetic field. Within the HVM approach, the present results can be employed to obtain a more realistic description of the KHI in the magnetopause. Indeed, the magnetic field across the magnetopause is neither exactly perpendicular to the plasma velocity nor exactly uniform, as assumed, for instance, in Settino et al. (2020). Beside an improved realism, the present solutions have the further advantage of being exactly stationary in comparison to, for instance, the approximate solutions by Cerri et al. (2013). This latter aspect can affect the development of the instability, as shown by Settino et al. (2020).

The outline of the paper is as follows. In Sect. 2, we present the physical model. In Sect. 3, we describe the singleparticle dynamics. In Sect. 4, we present a case with a uniform magnetic field, obliquely directed with respect to the bulk velocity. In Sect. 5, we discuss a case of uniform-intensity variable-direction magnetic field. Finally, in Sect. 6 we draw our conclusions.

\section{Equations of the model}

We consider a collisionless plasma formed by protons and electrons. The HVM model (e.g., Valentini et al. 2007) describes such a plasma at scales larger or on the order of proton scales (proton inertial length or Larmor radius, or both). In this model, protons are kinetically described by the particle DF of $f(\boldsymbol{x}, \boldsymbol{v}, t)$, where $\boldsymbol{x}$ and $\boldsymbol{v}$ are the position and the velocity coordinates in the phase-space, respectively, and $t$ is time. Electrons are treated as a massless fluid. Relevant moments of the proton DF are: the density, $n(\boldsymbol{x}, t)=\int f(\boldsymbol{x}, \boldsymbol{v}, t) d^{3} \boldsymbol{v}$, and bulk velocity, $\boldsymbol{u}(\boldsymbol{x}, t)=\int \boldsymbol{v} f(\boldsymbol{x}, \boldsymbol{v}, t) d^{3} \boldsymbol{v} / n(\boldsymbol{x}, t)$. Charge neutrality is assumed; therefore, the electron density is $n_{\mathrm{e}}=n$. The proton DF evolves in time according to the Vlasov equation:

$$
\frac{\partial f}{\partial t}+\boldsymbol{v} \cdot \nabla f+\frac{e}{m}\left(\boldsymbol{E}+\frac{\boldsymbol{v} \times \boldsymbol{B}}{c}\right) \cdot \frac{\partial f}{\partial \boldsymbol{v}}=0,
$$

where $e$ and $m$ are the proton charge and mass, $\boldsymbol{E}(\boldsymbol{x}, t)$ and $\boldsymbol{B}(\boldsymbol{x}, t)$ are the electric and magnetic fields, respectively, and $c$ is the speed of light. The set of equations includes Faraday's law, Ampere's law (where the displacement current is neglected), and a generalized Ohm's law:

$$
\frac{\partial \boldsymbol{B}}{\partial t}=-c \nabla \times \boldsymbol{E} ; \boldsymbol{j}=\frac{c}{4 \pi} \nabla \times \boldsymbol{B},
$$


$\boldsymbol{E}=-\frac{1}{c} \boldsymbol{u} \times \boldsymbol{B}+\frac{1}{e n}\left(\frac{\boldsymbol{j} \times \boldsymbol{B}}{c}\right)-\frac{1}{e n} \nabla p_{\mathrm{e}}$

where $\boldsymbol{j}$ is the current density and $p_{\mathrm{e}}$ is the electron pressure. In the HVM approach, it is often assumed that the electron fluid is isothermal, so that the electron pressure, $p_{\mathrm{e}}$, is a function only of the density, $n$. As we show further in this paper, for the present case, we need to discard the isothermal assumption and treat $p_{\mathrm{e}}$ as an independent quantity. Therefore a further equation is necessary to determine $p_{\mathrm{e}}$. Here we assume that the electron fluid is adiabatic and include the equation:

$\left[\frac{\partial}{\partial t}+\left(\boldsymbol{u}_{\mathrm{e}} \cdot \nabla\right)\right]\left(\frac{p_{\mathrm{e}}}{n^{\gamma_{\mathrm{e}}}}\right)=0$

where $\boldsymbol{u}_{\mathrm{e}}=\boldsymbol{u}-\boldsymbol{j} /($ en $)$ and $\gamma_{\mathrm{e}}$ are the electron bulk velocity and adiabatic index, respectively.

We look for a stationary solution of the system of Eqs. (1)-(4). If the electric and magnetic fields are constant in time, a DF expressed only in terms of single-particle constants of motion is a time-independent solution of the Vlasov Eq. (1). Therefore, to obtain a stationary solution we follow the following procedure: (a) starting from a specific form of $\boldsymbol{E}(\boldsymbol{x})$ and $\boldsymbol{B}(\boldsymbol{x})$, we first study the single particle motion, determining the constants of motions. (b) We look for a particular combination of such constants of motions that represents a DF, whose moments have a form close to the macroscopic structure we want to obtain. In particular, we require that the bulk velocity reproduces a planar shearing flow of the form $\boldsymbol{u}(x)=u(x) \boldsymbol{e}_{u}$, where $\boldsymbol{e}_{u}$ is a constant unit vector and the $x$ axis is perpendicular to $\boldsymbol{e}_{u}$. (c) Finally, calculating the moments of the DF we check a posteriori that the remaining Eqs. (2)-(4) are actually verified by the time-independent electromagnetic fields assumed at point (a). This procedure is the same as that used in Paper I for configurations different from those considered here.

\section{Single-particle motion}

We define a Cartesian reference frame $S=\{x, y, z\}$, where $x$ is the direction of the electric field while the magnetic field is parallel to $y z$ plane:

$\boldsymbol{E}=E(x) \boldsymbol{e}_{x} ; \quad \boldsymbol{B}=B_{0}\left\{\sin [\Phi(x)] \boldsymbol{e}_{y}+\cos [\Phi(x)] \boldsymbol{e}_{z}\right\}$,

with $B_{0}$ the uniform magnetic field intensity, $\Phi(x)$ the local angle between $\boldsymbol{B}$ and the $z$-axis, and $\boldsymbol{e}_{x}, \boldsymbol{e}_{y}$ and $\boldsymbol{e}_{z}$ unit vectors along the Cartesian axes. In our solution, the bulk velocity $\boldsymbol{u}=\boldsymbol{u}(x)$ is approximately directed along the $y$-axis. The two cases studied in Paper I correspond to $\Phi(x)=$ const $=0$ and $\Phi(x)=$ const $=\pi / 2, E(x)=0$. The electric field, $\boldsymbol{E}$, orthogonal to $\boldsymbol{B}$ produces a drift in the particle motion that is partially responsible for the bulk velocity, $\boldsymbol{u}$. In the particular case when $E(x)=$ const and $\Phi(x)=$ const, each particle drifts with a uniform drift velocity given by $\boldsymbol{v}_{d}=c \boldsymbol{E} \times \boldsymbol{B} / B^{2}$. Therefore, in regions where the bulk velocity is uniform (outside shear layers) the electric and magnetic field are both uniform. Finally, we observe that $\boldsymbol{j} \times \boldsymbol{B}=0$, where $\boldsymbol{j}$ is calculated through Ampere's law (2). Therefore, in the considered configuration no macroscopic Lorentz force is exerted on the plasma by the magnetic field.

The single-particle Lagrangian is given by

$\mathcal{L}(x, \boldsymbol{v}, t)=\frac{m}{2}\left(v_{x}^{2}+v_{y}^{2}+v_{z}^{2}\right)-e \phi\left(x ; x_{0}\right)+\frac{e B_{0}}{c}[\boldsymbol{a}(x) \cdot \boldsymbol{v}]$, where $\phi\left(x ; x_{0}\right)=-\int_{x_{0}}^{x} E\left(x^{\prime}\right) \mathrm{d} x^{\prime}$ is the electric potential, $x_{0}$ is a position where $\phi=0$, and $\boldsymbol{a}=a_{y}(x) \boldsymbol{e}_{y}+a_{z}(x) \boldsymbol{e}_{z}$ is a renormalized vector potential: $\boldsymbol{B}=\nabla \times\left(B_{0} \boldsymbol{a}\right)$. Since $y$ and $z$ are cyclic coordinates, two constant of motions are given by:

$w_{y}=v_{y}+\Omega_{p} a_{y}(x) ; w_{z}=v_{z}+\Omega_{p} a_{z}(x)$,

where $\Omega_{p}=e B_{0} /(m c)$ is the proton Larmor frequency. For given values of $w_{y}$ and $w_{z}$, relations (7) univocally express the velocity components $v_{y}$ and $v_{z}$ as functions of the particle position $x$. Writing the Lagrange equation $\mathrm{d}\left(\partial \mathcal{L} / \partial v_{x}\right) / \mathrm{d} t-(\partial \mathcal{L} / \partial x)=0$ and using relations (7) to eliminate $v_{y}$ and $v_{z}$, we obtain an equation for the particle motion along $x$ :

$$
\begin{aligned}
\frac{\mathrm{d} v_{x}}{\mathrm{~d} t}=-\frac{\mathrm{d}}{\mathrm{d} x}\left\{\frac{e}{m} \phi\left(x ; x_{0}\right)-\right. & \Omega_{p}\left[w_{y} a_{y}(x)+w_{z} a_{z}(x)\right] \\
& \left.+\frac{\Omega_{p}^{2}}{2}\left[a_{y}^{2}(x)+a_{z}^{2}(x)\right]\right\} .
\end{aligned}
$$

The LHS of Eq. (8) can be re-written in the form $\mathrm{d}\left(v_{x}^{2} / 2\right) / \mathrm{d} x$ and the equation can be integrated in the interval $\left[x_{0}, x\right]$. After computing the algebra, we obtain:

$\frac{1}{2} m v_{x}^{2}+U_{\mathrm{eff}}\left(x ; x_{0}\right)=e_{0}$,

where

$$
\begin{aligned}
U_{\mathrm{eff}}\left(x ; x_{0}\right)= & e \phi\left(x ; x_{0}\right)+\frac{1}{2} m \Omega_{p}^{2}\left[a_{y}(x)-a_{y}\left(x_{0}\right)\right]^{2} \\
& +\frac{1}{2} m \Omega_{p}^{2}\left[a_{z}(x)-a_{z}\left(x_{0}\right)\right]^{2} \\
& -m \Omega_{p} v_{0 y}\left[a_{y}(x)-a_{y}\left(x_{0}\right)\right]-m \Omega_{p} v_{0 z}\left[a_{z}(x)-a_{z}\left(x_{0}\right)\right],
\end{aligned}
$$

with $e_{0}=m v_{x 0}^{2} / 2$ a constant and $v_{0 i}=v_{i}\left(x=x_{0}\right), i=y, z$. Equation (9) expresses the energy conservation for a particle with mass $m$ following a $1 \mathrm{D}$ motion in the effective potential energy $U_{\text {eff }}\left(x ; x_{0}\right)$. In deriving the form in Eq. (10) we used Eq. (7), which is calculated at $x=x_{0}$ to express the constants $w_{y}$ and $w_{x}$ in terms of $v_{0 y}$ and $v_{0 z}$, respectively.

We want to describe a configuration where a finite number of shear layers are present, while far from that region the bulk velocity is uniform, corresponding to uniform $\boldsymbol{E}$ and $\boldsymbol{B}$. Therefore, for sufficiently large $|x|$ the scalar potential $\phi$ and the components $a_{y}$ and $a_{z}$ of the vector potential depend linearly on $x$ : $\phi\left(x ; x_{0}\right) \sim-E_{ \pm \infty} x+$ const; $a_{y}(x) \sim B_{z, \pm \infty} x / B_{0}+$ const; $a_{z}(x) \sim$ $-B_{y, \pm \infty} x / B_{0}+$ const, with $E_{ \pm \infty}, B_{y, \pm \infty}$ and $B_{z, \pm \infty}$ asymptotic values. Inspecting expression (10), it is clear that for sufficiently large $|x|$ the dominant terms in $U_{\text {eff }}\left(x ; x_{0}\right)$ are proportional to $x^{2}$. Hence, the particle motion along $x$ is confined within a potential well: $x_{m} \leq x \leq x_{M}$, where $U_{\text {eff }}\left(x_{m} ; x_{0}\right)=U_{\text {eff }}\left(x_{M} ; x_{0}\right)=e_{0}$ and the particle moves back and forth in the interval $\left[x_{m}, x_{M}\right]$, with vanishing $v_{x}$ at $x_{m}$ and $x_{M}$. In other words, $x(t)$ and $v_{x}(t)$ are periodic functions with a given period $\tau$, and then Eq. (7) implies that $v_{y}(t)$ and $v_{z}(t)$ are periodic with the same period $\tau$, too. Therefore, the particle follows a closed trajectory in the velocity space. In contrast, $y(t)$ and $z(t)$ are not necessarily periodic functions and the particle trajectory in the physical space is, in general, an open curve. We notice that such results are valid regardless of the specific field dependence on $x$, provided that the width of shear region is limited.

As a consequence of periodicity, the time-averaged velocity over the period $\tau$ provides the drift velocity in the particle 


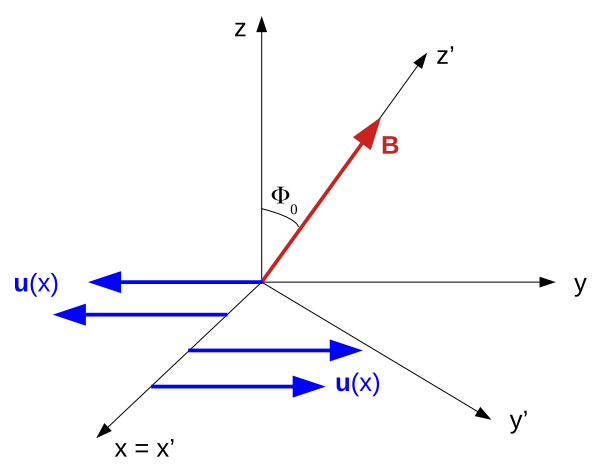

Fig. 1. Reference frames $S=\{x, y, z\}$ and $S^{\prime}=\left\{x^{\prime}, y^{\prime}, z^{\prime}\right\}$ are represented and the orientation of $\boldsymbol{B}$ and $\boldsymbol{u}$ are indicated for the uniform $\boldsymbol{B}$ configuration.

motion. Therefore, we define the guiding center $x$-position $x_{c}$ and velocity $\boldsymbol{v}_{c}$ as:

$x_{c}=\langle x\rangle_{c}=\frac{1}{\tau} \int_{0}^{\tau} x(t) \mathrm{d} t ; \quad \boldsymbol{v}_{c}=\langle\boldsymbol{v}\rangle_{\tau}=\frac{1}{\tau} \int_{0}^{\tau} \boldsymbol{v}(t) \mathrm{d} t$.

The periodicity of $x(t)$ implies $\left\langle v_{x}\right\rangle_{\tau}=0$; hence, the guiding center velocity has only two components $\boldsymbol{v}_{c}=v_{c y} \boldsymbol{e}_{y}+v_{c z} \boldsymbol{e}_{z}=$ $\left\langle v_{y}\right\rangle_{\tau} \boldsymbol{e}_{y}+\left\langle v_{z}\right\rangle_{\tau} \boldsymbol{e}_{z}$. Of course, $x_{c}, v_{c y}$, and $v_{c z}$ are constants of motion. In the particular case of uniform $\boldsymbol{B}$ (as in Paper I or in the next section), $a_{y}(x)$ and $a_{z}(x)$ are linear functions of $x$; as a consequence, the guiding center velocity $\boldsymbol{v}_{c}$ coincides with the projection of particle velocity onto the $y z$ plane, calculated at the guiding center position $x=x_{c}$. However, this latter property does not hold for a non-uniform $\boldsymbol{B}$. Finally, we notice that our definition (11) of guiding center is different from those used by Ganguli et al. (1988) and by Cai et al. (1990). In particular, the definition by Cai et al. (1990) implies that a particle can have more than one guiding center, while in our approach, a single guiding center is defined for each particle (see Paper I for a discussion).

Another constant of motion is given by the total energy $\mathcal{E}$, whose value depends on the choice of location $x_{0}$ where the electric potential $\phi\left(x ; x_{0}\right)$ vanishes. Although the value of $x_{0}$ does not affect the single particle motion, it is natural to choose $x_{0}=x_{c}$. In the case of a uniform electric and magnetic fields, such a choice is in accordance with the macroscopic invariance of the fluid properties with $x$ (see Paper I). Therefore, the total energy is defined by

$\mathcal{E}=K+e \phi\left(x ; x_{c}\right)=\frac{1}{2} m\left(v_{x}^{2}+v_{y}^{2}+v_{z}^{2}\right)+e \phi\left(x ; x_{c}\right)$,

where $K$ is the kinetic energy.

\section{Uniform oblique $B$}

We first consider a configuration corresponding to a uniform $\boldsymbol{B}$ obliquely oriented with respect to $\boldsymbol{u}(x)$. This kind of configuration has been considered in fluid models of KHI (e.g., Miura 1982) with applications to the magnetopause (Miura 1990). Indeed, the oblique- $\boldsymbol{B}$ configuration is more realistic for the magnetopause than a strictly perpendicular case (e.g., Settino et al. 2020). In the HVM framework, an approximately stationary state was obtained for this configuration by Cerri et al. (2013). In this work, we derive an exact stationary state.

The present case can be described by Eq. (5) when $\Phi(x)=$ $\Phi_{0}=$ const. We want to obtain a shearing flow where the bulk velocity $\boldsymbol{u}(x)$ is approximately directed along the $y$ direction and, therefore, forms an angle $\theta=\pi / 2-\Phi_{0}$ with the magnetic field. The present situation reduces to the "perpendicular" case studied in Paper I, when $\Phi_{0}=0$. In order to exploit this analogy, it is useful to introduce a rotated reference frame $S^{\prime}=\left\{x^{\prime}, y^{\prime}, z^{\prime}\right\}$, where $x^{\prime}$ and $x$ axes coincide and $z^{\prime}$ axis is parallel to $\boldsymbol{B}$. The two reference frames and other quantities are illustrated in Fig. 1. In this configuration no force is acting along $z^{\prime}$ and therefore the particle velocity component $v_{z^{\prime}}$ is a constant of motion. As a consequence, the "perpendicular" energy $\mathcal{E}_{\perp}=\mathcal{E}-m v_{z^{\prime}}^{2} / 2$ is a constant of motion as well.

Let us consider first the DF of the perpendicular case of Paper I (Eq. (31) of Paper I) corresponding to the case $\Phi_{0}=0$ :

$f_{\perp}\left(x, v_{x}, v_{y}, v_{z}\right)=C \exp \left[-\frac{\mathcal{E}_{0}\left(x, v_{x}, v_{y}, v_{z}\right)}{m v_{t h, p}^{2}}\right]$,

where $\mathcal{E}_{0}=\mathcal{E}-m v_{c \perp}^{2} / 2$ is the "reduced" total energy where the kinetic energy associated with the guiding center motion has been subtracted, and $v_{c \perp}$ is the guiding center velocity component perpendicular to $\boldsymbol{B}$ (the only non-vanishing component in this case). $C$ is a normalization constant and $v_{\text {th,p }}$ is the proton thermal speed away from the shear region, where $f_{\perp}$ is reduced to an SM. The associated bulk velocity $\boldsymbol{u}_{\perp}$ is also directed along the $y^{\prime}$ direction (Paper I).

In the oblique case $\left(\Phi_{0} \neq 0\right)$, we want to obtain a bulk velocity $\boldsymbol{u}$ in the $y$ direction, which has two components: $\boldsymbol{u}_{\perp}$ and $\boldsymbol{u}_{\|}$, respectively, which are perpendicular and parallel to $\boldsymbol{B}$. As in Paper I, the former component is due to the guiding center velocity $v_{c \perp}$ of particles. To obtain a parallel bulk velocity, $\boldsymbol{u}_{\|}$, as well, we add a shift $U_{\|}$of the DF in the velocity space along the $v_{z^{\prime}}$ direction, similar to the shift that transforms a Maxwellian into an SM. This is coherent with the cyclicity of the $z^{\prime}$ coordinate. The amount $U_{\|}$of the shift should (roughly) correspond to guiding centers moving along the $y$ direction. The geometry suggests the relation $U_{\|} / v_{c \perp}=\tan \Phi_{0}$. On the basis of the above considerations, we modify the expression (13) and write the following form for the DF of uniform oblique $\boldsymbol{B}$ :

$$
\begin{aligned}
f_{\Phi_{0}}(x, \boldsymbol{v})= & C \exp \left\{-\frac{1}{2 v_{\mathrm{th}, \mathrm{p}}^{2}}\left[v_{x}^{2}+\left[v_{y^{\prime}}\left(v_{y}, v_{z}, \Phi_{0}\right)\right]^{2}\right.\right. \\
& +\left[v_{z^{\prime}}\left(v_{y}, v_{z}, \Phi_{0}\right)-v_{c \perp}(x, \boldsymbol{v}) \tan \Phi_{0}\right]^{2} \\
& \left.\left.+(2 e / m) \phi\left(x ; x_{c}(x, \boldsymbol{v})\right)-\left[v_{c, \perp}(x, \boldsymbol{v})\right]^{2}\right]\right\},
\end{aligned}
$$

where $v_{y^{\prime}}\left(v_{y}, v_{z}, \Phi_{0}\right)=v_{y} \cos \Phi_{0}-v_{z} \sin \Phi_{0}, v_{z^{\prime}}\left(v_{y}, v_{z}, \Phi_{0}\right)=$ $v_{y} \sin \Phi_{0}+v_{z} \cos \Phi_{0}$, and $v_{c, \perp}(x, \boldsymbol{v})$ is the guiding center velocity of a particle that at a given time is at position $x$ with velocity $v$ (the knowledge of $x$ and $v$ fully determines the particle motion). The particular dependence of the DF (14) on the parallel drift velocity component $v_{z^{\prime}}$ allows us to counterbalance the $z$ component of the averaged gyrocenter velocity, thus obtaining a bulk velocity nearly aligned with the $y$ direction. We observe that the form (14) for $\Phi_{0}=0$ is reduced to the distribution function obtained in the case of "perpendicular B" (Eq. (31) of Paper I). It is important to note that the DF defined by Eq. (14) is expressed only in terms of constants of motion. In fact, the terms $v_{x}^{2}+v_{y^{\prime}}^{2}+(2 e / m) \phi\left(x ; x_{c}\right)=2 \mathcal{E}_{\perp} / m, v_{z^{\prime}}$, and $v_{c \perp}$ are all constants of motions. Therefore, the DF (14) is a stationary solution of the Vlasov equation, provided that $\boldsymbol{E}$ and $\boldsymbol{B}$ remain stationary. This latter condition is to be subsequently verified.

The form (14) has been heuristically induced in the attempt to generalize the case considered in Paper I. Therefore, the associated bulk velocity, $\boldsymbol{u}$, must be calculated a posteriori in order 
to verify to what extent it has the expected form. We also notice that the form (14) only implicitly defines the DF, because explicit expressions for the guiding center velocity $v_{c \perp}(x, \boldsymbol{v})$ and position $x_{c}(x, v)$ are not known for an arbitrary electric field profile $E(x)$. Explicit analytical results will be obtained in the particular case of a linear profile for the electric field, denoted as a "local approximation". In the general case, a numerical method will be applied to calculate the DF and its moments.

\subsection{Local approximation}

In the particular case of an electric field with a linear profile the single particle motion can be analytically calculated. In this case, the form (14) of the DF and of its moments can be explicitly written. In the following we briefly describe the main results of this case, while more details are given in Appendix A. The electric field has the form:

$E(x)=E_{0}+\alpha_{0}\left(x-x_{0}\right)$,

with $E_{0}$ and $\alpha_{0}$ constant. Equation (15) can also be interpreted as a local first-order approximation of a general profile if $\left|x-x_{0}\right| \ll\left|E_{0} / \alpha_{0}\right|$. For $\alpha_{0} \geq m \Omega_{p}^{2} / e$ particles moves along $x$ linearly or exponentially in time, leading to a breakdown of the local approximation. Therefore, we consider only the case $\alpha_{0}<$ $m \Omega_{p}^{2} / e$, when the particle orbit in the velocity space is given by a shifted ellipse located in a plane parallel to the $v_{x^{\prime}} v_{y^{\prime}}$ plane, which is traveled with a gyrofrequency $\omega=\left(\Omega_{p}^{2}-e \alpha_{0} / m\right)^{1 / 2}$. The guiding center is the ellipse center (the explicit expression of $x_{c}(x, v)$ is given in Appendix A); it moves with a transverse velocity that coincides with the $\boldsymbol{E} \times \boldsymbol{B}$ drift velocity calculated at the guiding center position: $v_{c \perp}(x, \boldsymbol{v})=-c E\left(x_{c}\right) / B$. A simple physical interpretation can be given to the condition $\alpha_{0}<m \Omega_{p}^{2} / e$. In fact, such a condition corresponds to $\Delta F_{e} \lesssim F_{M}$, where $\Delta F_{e}=e \alpha_{0} \rho \sim e \alpha_{0} v / \Omega_{p}$ is the electric force variation across a length equal to the Larmor radius $\rho$, while $v$ and $F_{M} \sim m v \Omega_{p}$ are estimations of the particle velocity and of the magnetic force, respectively. Therefore, in the local approximation the particle trajectory is bounded provided that the magnetic force, responsible for the gyromotion, is large enough to hinder particle runaway due to $F_{e}$ on a scale comparable with the Larmor radius. Using the expressions of $x_{c}(x, \boldsymbol{v})$ and $v_{c \perp}(x, \boldsymbol{v})$, the explicit form of the DF can be derived:

$$
\begin{aligned}
f_{\Phi_{0}}^{(l a)}(x, \boldsymbol{v})= & \frac{n_{0}}{(2 \pi)^{3 / 2} v_{\mathrm{th}, \mathrm{p}}^{3}} \sqrt{\frac{\Omega_{p} B_{0}}{\Omega_{p} B_{0}-c \alpha_{0}}} \exp \left\{-\frac{1}{2 v_{\mathrm{th}, \mathrm{p}}^{2}}\right. \\
& \times\left\{v_{x}^{2}+\frac{\Omega_{p} B_{0}}{\Omega_{p} B_{0}-c \alpha_{0}}\left[\frac{c \alpha_{0}}{B_{0}}\left(x-x_{0}\right)+v_{y^{\prime}}-v_{d 0}\right]^{2}\right. \\
& +\left[v_{z^{\prime}}-v_{d 0} \tan \Phi_{0}+\frac{c \alpha_{0} \tan \Phi_{0}}{\Omega_{p} B_{0}-c \alpha_{0}}\right. \\
& \left.\left.\left.\times\left[\Omega_{p}\left(x-x_{0}\right)+v_{y^{\prime}}-v_{d 0}\right]\right]^{2}\right\}\right\},
\end{aligned}
$$

where $n=n_{0}$ is the uniform density and $v_{d 0}=-c E_{0} / B_{0}$ is the drift velocity at the position $x_{0}$. The associated bulk velocity takes the form:

$\boldsymbol{u}(\boldsymbol{x})=-\frac{c E(x)}{B_{0}} \boldsymbol{e}_{y^{\prime}}-\frac{c E(x)}{B_{0}} \tan \Phi_{0} \boldsymbol{e}_{z^{\prime}}=-\frac{c E(x)}{B_{0} \cos \Phi_{0}} \boldsymbol{e}_{y}$.

The expression (17) corresponds to a planar shearing flow varying along $x$ and directed at constant angle $\theta=\pi / 2-\Phi_{0}$ with $\boldsymbol{B}$, at each $x$. Therefore, in the local approximation, the DF (14) reproduces the desired form for the bulk velocity exactly. In particular, the bulk velocity component, $u_{\perp}=u_{y^{\prime}}$, that is perpendicular to $\boldsymbol{B}$ is equal to the local $\boldsymbol{E} \times \boldsymbol{B}$ drift velocity.

\subsection{General electric field profile}

In the case of an electric field with a general profile, $E(x)$, we used a numerical procedure to calculate the DF (14) and its moments. In the procedure, where the motion equations of single particles are integrated, the periodicity in the velocity space represents a key element. Of course, constants of motions appearing in expression (14) can be evaluated at any instant of time; therefore, the phase-space coordinates $\{x, \boldsymbol{v}\}$ are interpreted as the particle position and velocity at the initial time of its motion. In the numerical procedure, such initial conditions are taken on a regular grid in the phase-space (1D in the physical space and $3 \mathrm{D}$ in the velocity space): $x(t=0)=x_{i}, v_{x}(t=0)=v_{x, j}$, $v_{y}(t=0)=v_{y, k}, v_{z}(t=0)=v_{z, l}(i, j, k, l$ are indexes that span on the 4D grid and identify the given particle). Starting from this set of initial conditions, the motion equations of each particle are integrated during one single period $\tau_{i j k l}$. For the time integration, we used a third-order Adams-Bashforth scheme. Then the constants of motion were calculated: the guiding center transverse velocity $v_{c \perp, i j k l}=\left\langle v_{\perp}\right\rangle_{\tau_{i j k l}}$ and position $x_{c, i j k l}=\langle x\rangle_{\tau_{i j k l}}$, as well as the transverse energy $U_{\perp, i j k l}=m\left(v_{y, k}^{2}+v_{z, l}^{2}\right) / 2-e \int_{x_{c, i j k l}}^{x_{i}} E(x) d x$. Finally, these quantities, along with the parallel velocity $v_{z^{\prime}, k l}=$ $v_{y, k} \cos \Phi_{0}-v_{z, l} \sin \Phi_{0}$, are inserted into expression (14) and the value of $f_{\phi_{0}}\left(x_{i}, v_{x, j}, v_{y, k}, v_{z, l}\right)$ for the $\mathrm{DF}$ at the given phase-space point is calculated.

The set of values of the DF calculated on the grid points allow us to obtain the moments by numerically calculating the corresponding integrals. In particular, we found that the $x$ component of the resulting bulk velocity is vanishing: $u_{x}=0$, similar as in the local approximation (Eq. (17)). Therefore, the term $\boldsymbol{u} \times \boldsymbol{B}$ in the generalized Ohm's law (3) is directed in the $x$ direction. Moreover, in this configuration it is $\boldsymbol{j}=0$. Hence, only the $x$ component of Eq. (3) is nonvanishing, which implies:

$$
\frac{\mathrm{d} p_{e}}{\mathrm{~d} x}=-e n(x)\left\{E(x)+\frac{B_{0}}{c}\left[u_{y}(x) \cos \Phi_{0}-u_{z}(x) \sin \Phi_{0}\right]\right\} .
$$

This equation determines the electron pressure $p_{e}(x)$.

Concerning the time dependence, let us assume that the obtained configuration holds at the initial time. Since $\partial f_{\Phi_{0}} / \partial t=$ 0 , all the moments of $f_{\Phi_{0}}$ have a vanishing time derivative; in particular, it is $\partial n / \partial t=0$. Being $\nabla \times \boldsymbol{E}=0$, the Faraday's law (2) implies that $\partial \boldsymbol{B} / \partial t=0$. Moreover, being $(\boldsymbol{u} \cdot \nabla)\left(p_{\mathrm{e}} / n^{\gamma_{\mathrm{e}}}\right)=0$, Eq. (4) implies $\partial p_{\mathrm{e}} / \partial t=0$. Hence, from the Ohm's law (3), it follows that $\partial \boldsymbol{E} / \partial t=0$. We can conclude that the considered configuration is a stationary solution of the full set of HVM Eqs. (1)-(4).

\subsection{Results for a double shear configuration}

To illustrate our results, we consider a particular configuration with two opposite shear layers. We introduce rescaled variables: the magnetic field is normalized to $B_{0}$, time to $\Omega_{p}^{-1}$, density to the value $\bar{n}$ away from the shear regions, velocities to the Alfvén velocity $v_{A}=B_{0} /(4 \pi m \bar{n})^{1 / 2}$, lengths to the proton inertial length $d_{p}=v_{A} / \Omega_{p}$, electric field to $v_{A} B_{0} / c$, and electron pressure to $\bar{n} m v_{A}^{2}$. To simplify the notation, in the remainder of this subsection, we denote rescaled quantities with the same symbols as the original quantities. In the homogeneous region, the proton plasma beta is $\beta=2 v_{\text {th,p }}^{2} / v_{A}^{2}=2$. We chose a spatial domain, $D$, defined by $0 \leq x \leq L$, with $L=100$ and the following form for 


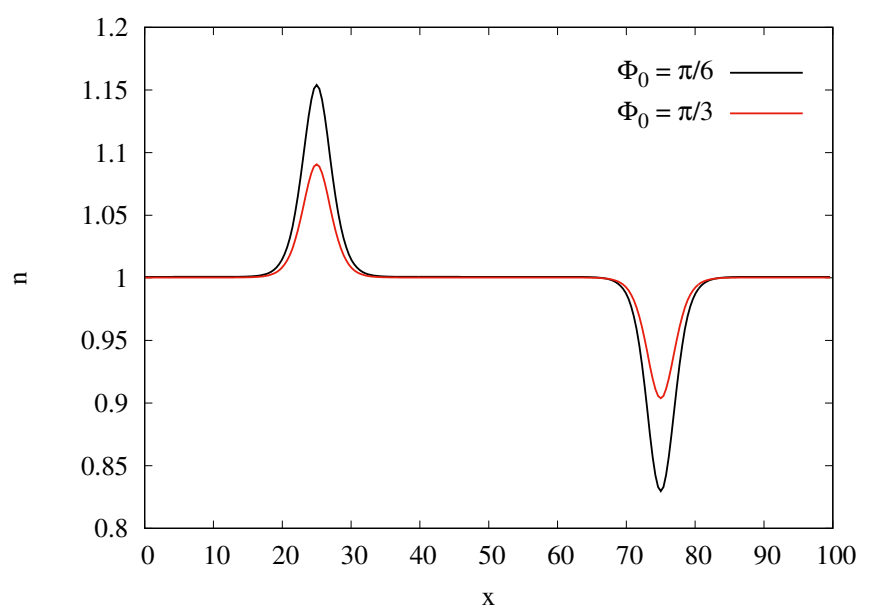

Fig. 2. Profiles of density $n(x)$ calculated for $\Phi_{0}=\pi / 6$ (black line) and $\Phi_{0}=\pi / 3$ (red line) in the uniform oblique $\boldsymbol{B}$ case.

the electric field:

$E(x)=E_{0}\left[1-\tanh \left(\frac{x-L / 4}{\Delta x}\right)+\tanh \left(\frac{x-3 L / 4}{\Delta x}\right)\right]$,

where $E_{0}=\cos \Phi_{0}$ and $\Delta x=2.5$. This corresponds to two opposite shear layers of a width, $\Delta x$, located at $x=L / 4$ and $x=3 L / 4$, respectively. The proton thermal gyroradius in normalized units is $\rho=v_{\text {th }, \mathrm{p}} / \Omega_{p}=1$, therefore, the shear width $\Delta x$ is 2.5 larger than $\rho$. The electric field (19) is periodic in the domain $D: E(0)=E(L) \simeq E_{0}$, while in the center of the domain it is $E(L / 2) \simeq-E_{0}$. Moreover, $E(x)$ vanishes in the limit $\Phi_{0} \rightarrow \pi / 2$, consistent with the case when $\boldsymbol{B}$ is parallel to $\boldsymbol{u}$ (Paper I). We calculated the DF (14) using the above-described procedure on a grid formed by $N_{x}=256$ points in the $x$ direction and $N_{v}=71$ points in each velocity direction, $v_{i}, i=x, y, z$, where $-7 v_{\text {th, }} \leq v_{i} \leq 7 v_{\text {th, }}$, with $v_{\text {th, }}=1$ (in scaled units). The $N_{x} N_{v}^{3} \simeq 9.16 \times 10^{7}$ particle trajectories have been calculated using a parallel computing procedure.

The associated density $n(x)=\int f_{\Phi_{0}}(x, \boldsymbol{v}) d^{3} \boldsymbol{v}$ is plotted in Fig. 2 for cases when $\Phi_{0}=\pi / 6$ and $\Phi_{0}=\pi / 3$. In contrast with the local approximation, now the density, $n(x)$, is not uniform, except away from the two shear regions. The non-uniformity of $n(x)$ decreases with increasing $\Phi_{0}$; actually, when $\boldsymbol{B}$ is parallel to $\boldsymbol{u}$ (corresponding to $\Phi_{0}=\pi / 2$ ) the density profile is uniform (Paper I). In particular, $n(x)$ is maximum (minimum) at the center of the shear at $x=L / 4(x=3 L / 4)$. This asymmetry between the two shear layers is a genuine kinetic effect that is related to the sign of the dot product $(\nabla \times \boldsymbol{u}) \cdot \boldsymbol{\Omega}_{p},\left(\boldsymbol{\Omega}_{p}\right.$ being the proton vector gyration angular velocity) which is opposite in the two shear layers.

The profiles of the bulk velocity components $u_{i}(x)=$ $\int v_{i} f_{\Phi_{0}}(x, \boldsymbol{v}) d^{3} \boldsymbol{v} / n(x), i=y, z$ are plotted in Fig. 3 for $\Phi_{0}=\pi / 6$. We notice that $\boldsymbol{u}$ is not exactly in the $y$ direction, as in the local approximation case. However, $\left|u_{z}\right|$ is about two orders of magnitude smaller than $\left|u_{y}\right|$, while $\left|u_{x}\right| \sim 10^{-12}$ (not shown). Therefore, the condition that $\boldsymbol{u}(x)$ and $\boldsymbol{B}$ form a constant angle $\theta=\left(\pi / 2-\Phi_{0}\right)$ is well satisfied. The largest deviations from this condition are localized at the shear layers. Moreover, the profile of $u_{z}(x)$ is qualitatively similar to $-d^{2} E(x) / \mathrm{d} x^{2}$; this is not surprising, since for a linear electric field profile (15), it is $u_{z}(x)=0$.

In the shear regions, the DF deviates from a Maxwellian. In particular, a temperature anisotropy develops that can be quantitatively evaluated by calculating the variance matrix. This has

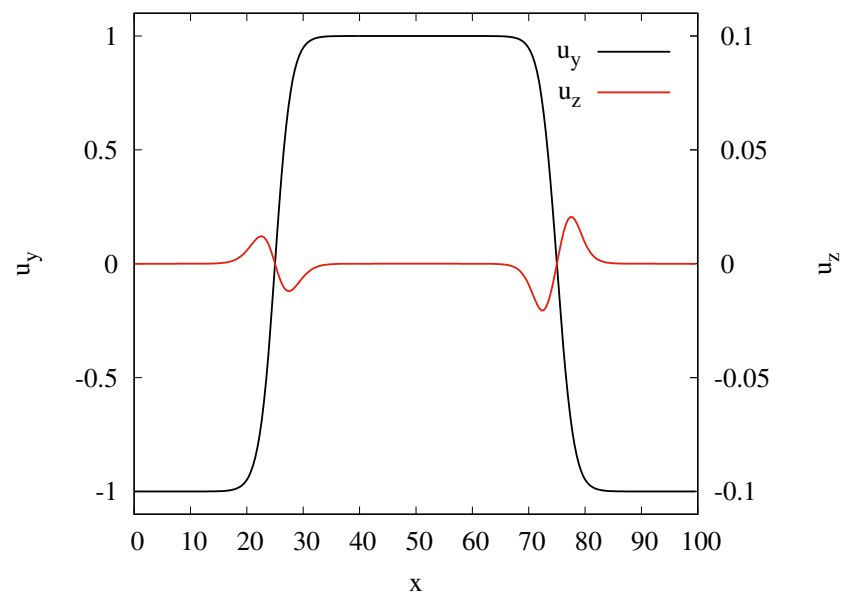

Fig. 3. Profiles of bulk velocity components $u_{y}(x)$ (black line) and $u_{z}(x)$ (red line) calculated for $\Phi_{0}=\pi / 6$ in the uniform oblique $\boldsymbol{B}$ case. Different scales are used for $u_{y}$ and $u_{z}$.

been done in the reference frame $S^{\prime}$ where $\boldsymbol{B}$ is along the $z^{\prime}$ axis. We refer to $S^{\prime}$ as "local $\boldsymbol{B}$ frame" (LBF). The corresponding variance matrix is

$\sigma_{i j}^{\mathrm{LBF}}(x)=\frac{1}{n(x)} \int\left[v_{i}-u_{i}(x)\right]\left[v_{j}-u_{j}(x)\right] f_{\Phi_{0}}(x, \boldsymbol{v}) d^{3} \boldsymbol{v}$,

with $i, j=x^{\prime}, y^{\prime}, z^{\prime}$. Normalized parallel and perpendicular temperatures are given by $T_{\|}=\sigma_{z^{\prime} z^{\prime}}^{L B F}$ and $T_{\perp}=\left(\sigma_{x^{\prime} x^{\prime}}^{L B F}+\sigma_{y^{\prime} y^{\prime}}^{L B F}\right) / 2$, respectively, while the anisotropy and agyrotropy indexes are $\eta^{*}=T_{\perp} / T_{\|}$and $\zeta^{*}=\min \left(\sigma_{x^{\prime} x^{\prime}}^{\mathrm{LBF}}, \sigma_{y^{\prime} y^{\prime}}^{\mathrm{LBF}}\right) / \max \left(\sigma_{x^{\prime} x^{\prime}}^{\mathrm{LBF}}, \sigma_{y^{\prime} y^{\prime}}^{\mathrm{LBF}}\right)$, respectively (a gyrotropic DF corresponds to $\zeta^{*}=1$, otherwise it is $\left.\zeta^{*}<1\right)$. Since the variance matrix is symmetric, a reference frame exists (denoted as the minimum variance frame, MVF) where it is diagonal. Therefore, the eigenvalues $\lambda^{(3)}<\lambda^{(2)}<\lambda^{(1)}$ of $\sigma_{i j}$ give the temperatures along the MVF axes. The corresponding anisotropy and agyrotropy indexes are defined by $\eta=\left(\lambda^{(2)}+\lambda^{(3)}\right) /\left(2 \lambda^{(1)}\right)$ and $\zeta=\lambda^{(3)} / \lambda^{(2)}$, respectively. In Fig. 4 , the profiles of $\eta, \eta^{*}, \zeta$, and $\zeta^{*}$ are plotted as functions of $x$, for $\Phi_{0}=\pi / 6$. In the shear layers, the DF is both anisotropic and agyrotropic. Anisotropy is larger in the MVF than in the LBF in both shear layers. Agyrotropy is larger in the LBF than in the MVF in the shear layer at $x=L / 4$, the reverse holds at $x=3 L / 4$, owing to a different orientation between $\boldsymbol{\Omega}_{p}$ and $\nabla \times \boldsymbol{u}$ at each shear. A similar behavior holds also for $\phi_{0}=\pi / 3$, except for a larger agyrotropy in the MVF in both shears (not shown).

Finally, we calculated the heat flux $\boldsymbol{q}$, defined by

$\boldsymbol{q}(x)=\frac{1}{2} \int[\boldsymbol{v}-\boldsymbol{u}(x)]|[\boldsymbol{v}-\boldsymbol{u}(x)]|^{2} f_{\Phi_{0}}(x, \boldsymbol{v}) d^{3} \boldsymbol{v}$.

The components $q_{y}$ and $q_{z}$ are plotted in Fig. 5 as functions of $x$, for $\Phi_{0}=\pi / 6$ and $\Phi_{0}=\pi / 3$, while $q_{x}$ is null for all $x$ values. We see that $q_{y}$ and $q_{z}$ are not vanishing in the shear layers, where they have opposite behaviors. However, the average of both $q_{y}$ and $q_{z}$ across each shear layer is null.

\subsection{Numerical simulation}

Although the above-described configuration is, in principle, exactly stationary, since it has been derived through a numerical procedure it is necessarily affected by errors that arise during the time integration of single particle motion. Moreover, in the perspective of applying such results in numerical simulations, 

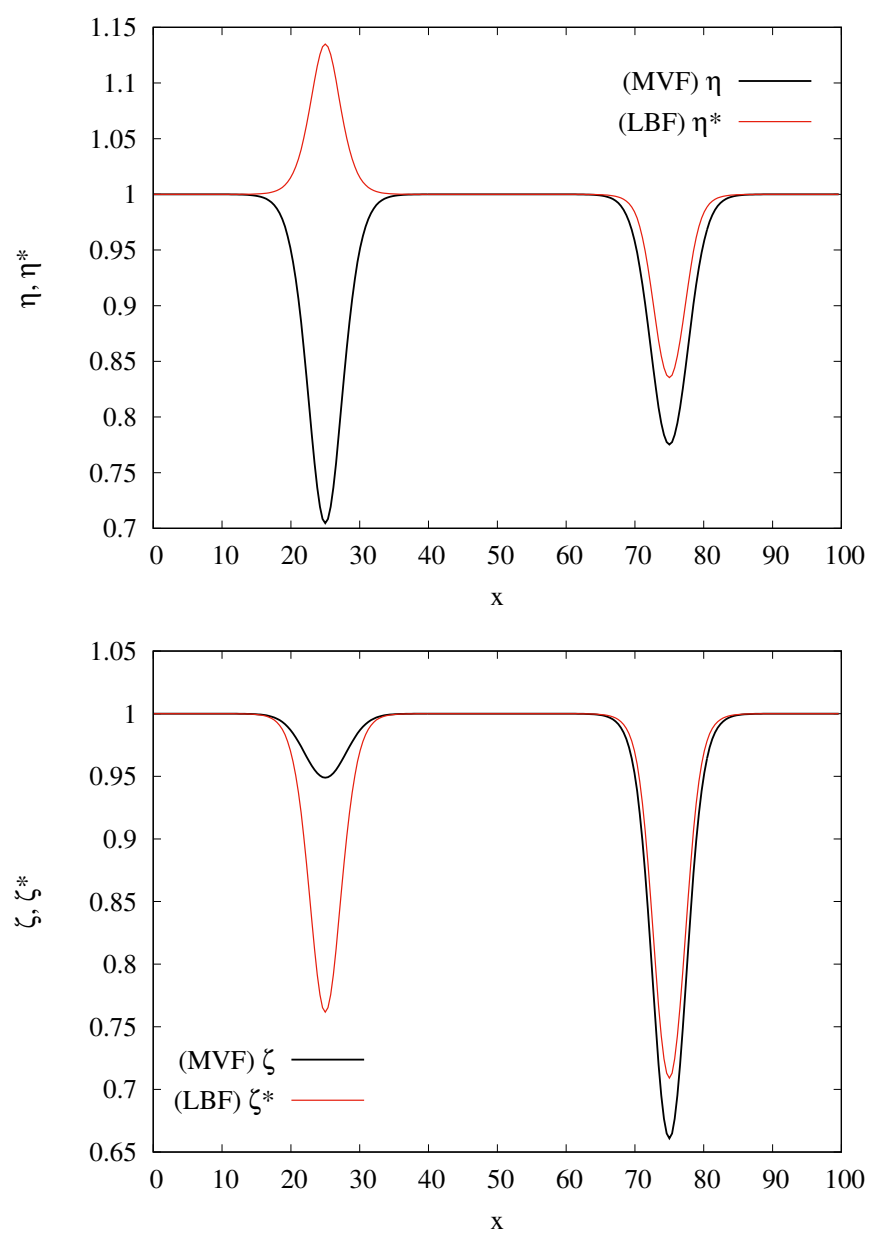

Fig. 4. Upper panel: profiles of anisotropy parameters $\eta$ (black line) and $\eta^{*}$ (red line); lower panel: profiles of agyrotropy parameters $\zeta$ (black line) and $\zeta^{*}$ (red line). Both panels refer to $\Phi_{0}=\pi / 6$ in the uniform oblique $\boldsymbol{B}$ case.

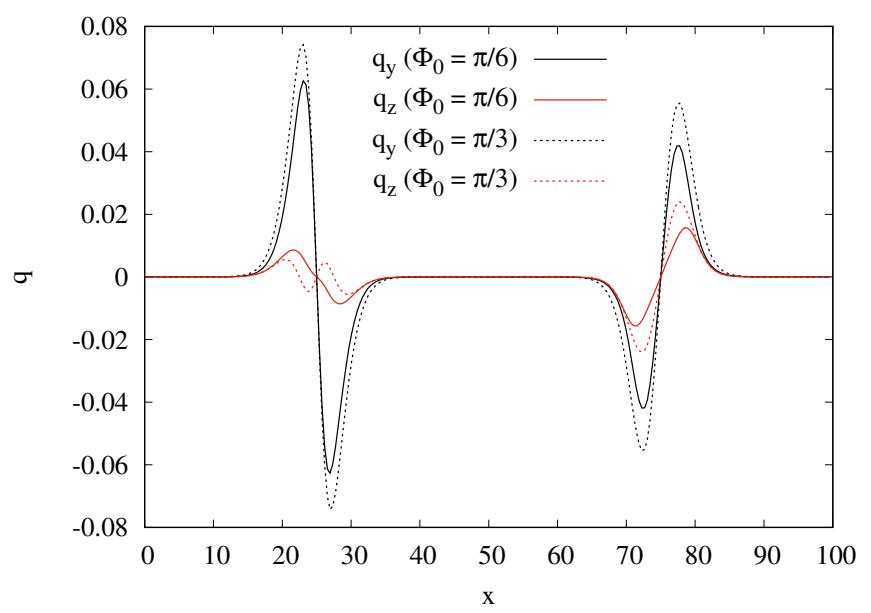

Fig. 5. Profiles of heat flux components $q_{y}$ (black lines) and $q_{z}$ (red lines) for $\Phi_{0}=\pi / 6$ (full lines) and $\Phi_{0}=\pi / 3$ (dashed lines) in the uniform oblique $\boldsymbol{B}$ case.

other errors would appear related to discretization in the phase space and time that is inherent to numerical codes. Therefore, it is interesting to study the time behavior of the above-described stationary state when it is used as initial condition in a HVM

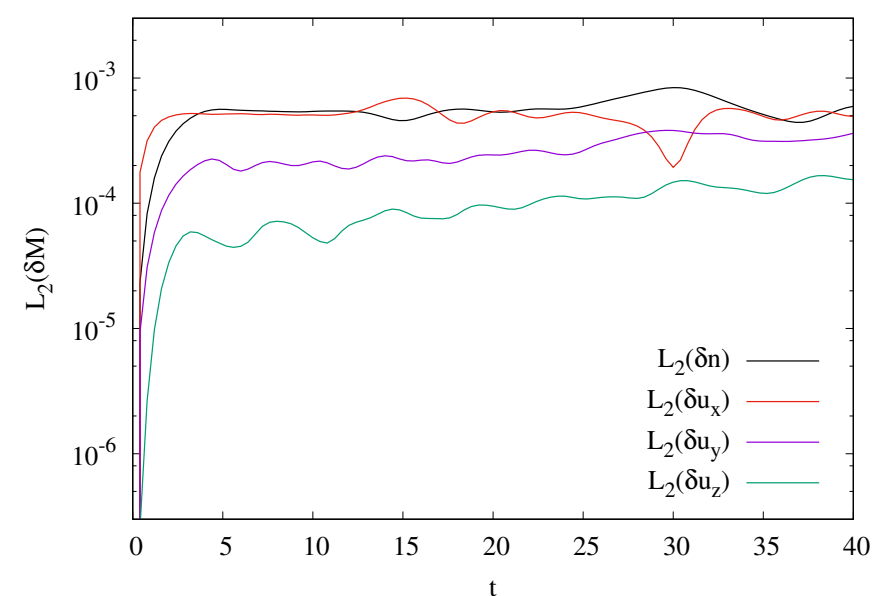

Fig. 6. Profiles of $L_{2}(\delta n)$ (black line), $L_{2}\left(\delta u_{x}\right)$ (red line), $L_{2}\left(\delta u_{y}\right)$ (purple line), and $L_{2}\left(\delta u_{z}\right)$ (green line) as functions of time, as they result from the HVM simulation.

numerical simulation. This has the purpose to find a quantitative evaluation of departure from stationarity due to numerical errors.

A simulation was performed using a HVM numerical code, where periodic boundary conditions are applied in the physical space while it is assumed that the DF vanishes at the boundaries of the domain in the velocity space $\left(\left|v_{x, y, z}\right| \geq v_{\max }=7 v_{\text {th,p }}\right)$. Details on the code can be found in Valentini et al. (2007). The simulation lasts up to the time $t=40$ in normalized units. We calculated the time evolution of density and bulk velocity components, evaluating the departures from their initial profiles. In particular, for a given moment $M(x, t)$, we calculated the corresponding $L_{2}$-norm departure with respect to the initial profile $M(x, 0)$ :

$L_{2}(\delta M)(t)=\left\{\frac{1}{L} \int_{0}^{L}[M(x, t)-M(x, 0)]^{2} d x\right\}^{1 / 2}$.

In Fig. 6 , the density $L_{2}(\delta n)$ and bulk velocity $L_{2}\left(\delta u_{i}\right)(i=x, y, z)$ departures are plotted as functions of $t$, as they result from the simulation. As expected, such departures do not remain null. However, after a fast increase lasting few time units, both $L_{2}(\delta n)$ and $L_{2}\left(\delta u_{i}\right)$ saturate at a level that has an order of magnitude between $10^{-4}$ and $10^{-3}$. In Fig. 7 the profiles $u_{y}(x)$ calculated at the initial and at the final time are plotted. The two profiles are practically superimposed; therefore, the width of shear layers remains essentially unchanged during the considered time evolution. To visualize variations of the profile $u_{y}(x)$ during the time evolution; in the same figure, we superimposed the difference, $\Delta u_{y}(x)=u_{y}(x, t=40)-u_{y}(x, t=0)$ (using a different scale). It can be seen that $\max \left|\Delta u_{y}\right|$ is of the order of 0.001 and variations are essentially confined within the two shear layers. We conclude that numerical errors affect the stationarity of the solution to a low extent.

\section{B with uniform intensity and variable direction}

The second configuration, corresponds to a nonuniform magnetic field, $\boldsymbol{B}$, whose direction changes in space, but keeping $|\boldsymbol{B}|$ uniform. This kind of configuration is more realistic for the magnetopause than the previous case where $\boldsymbol{B}$ had a constant direction. In fact, in many circumstances the magnetic field direction on the solar wind side is different from that on the magnetosphere side (see, e.g., Faganello \& Califano 2017). The present 


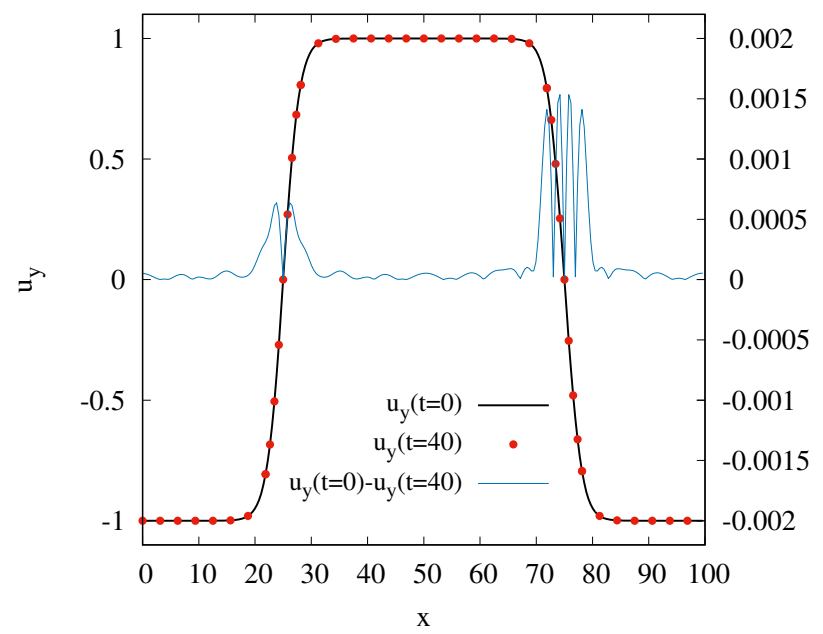

Fig. 7. Profiles of $u_{y}(x)$ calculated at the initial (black solid line) and final (red balls) time of the simulation. The difference between the two curves $\Delta u_{y}$ is also plotted (blue solid line).

case corresponds to a configuration described by Eq. (5), where the angle $\Phi(x)$ between $\boldsymbol{B}(x)$ and the $z$-axis is not constant. In this case, particle trajectories are not planar curves.

To have an indication to how to find an appropriate stationary solution, we first examined a local approximation. We consider electric and magnetic field varying linearly with $x$, assuming the form (15) for $E(x)$ and the equations:

$B_{y}(x)=B_{0}\left[b_{0 y}+\gamma_{y}\left(x-x_{0}\right)\right]$,

$B_{z}(x)=B_{0}\left[b_{0 z}-\gamma_{z}\left(x-x_{0}\right)\right]$,

where $b_{0 y, 0 z}$ and $\gamma_{y, z}$ are constants and $\left|x-x_{0}\right| \ll\left|b_{0 y, 0 z} / \gamma_{y, z}\right|$. In studying the particle dynamics, all terms that are quadratic or of higher order in the ratios $\left|\gamma_{y, z}\left(x-x_{0}\right) / b_{0 y, 0 z}\right|$ will be neglected. Here, we give only the main results of the procedure, while details can be found in the Appendix B. The particle motion along $x$ corresponds to a harmonic oscillator $x(t)=R_{0} \sin (\omega t+$ $\varphi)+x_{c}$, where the $R_{0}$ is amplitude, $\varphi$ is the phase, the frequency is

$\omega=\left[\Omega_{p}^{2}+\Omega_{p}\left(w_{y} \gamma_{z}+w_{z} \gamma_{y}\right)-\frac{e \alpha_{0}}{m}\right]^{1 / 2}$,

and $x_{c}$ is the center of the oscillation, corresponding to the guiding center position (the expression of $x_{c}$ is given in Appendix B). This solution holds if $\alpha_{0}<(m / e)\left[\Omega_{p}^{2}+\Omega_{p}\left(w_{y} \gamma_{z}+w_{z} \gamma_{y}\right)\right]$, otherwise the local approximation breaks down. Coordinates $y(t)$ and $z(t)$ can be calculated using the Eq. (7). Due to the quadratic form of $a_{y}(x)$ and $a_{z}(x)$, the coordinates $y(t)$ and $z(t)$ as well as the velocity components $v_{y}(t)$ and $v_{z}(t)$ are no longer harmonic functions of time. This implies that the closed trajectory in the velocity space is not an ellipse, as in the previous configuration and, in general, it is not planar. However, the following simple relation holds for the components $v_{c y}=\left\langle v_{y}\right\rangle$ and $v_{c z}=\left\langle v_{z}\right\rangle$ of the guiding center velocity: $v_{c z} B_{y}\left(x_{c}\right)-v_{c y} B_{z}\left(x_{c}\right)=c E\left(x_{c}\right)$, which implies that the guiding center velocity component perpendicular to $\boldsymbol{B}\left(x_{c}\right)$ is equal to the local $\boldsymbol{E} \times \boldsymbol{B}$ drift velocity:

$v_{c \perp}=c \frac{\boldsymbol{E}\left(x_{c}\right) \times \boldsymbol{B}\left(x_{c}\right)}{B_{0}^{2}}$.

This same property of the guiding center velocity was found in the oblique $\boldsymbol{B}$ configuration. This analogy will be exploited to generalize the DF found in the case of oblique uniform $\boldsymbol{B}$ to the present case of non-uniform $\boldsymbol{B}$. For this purpose, we go back to the uniform- $\boldsymbol{B}$ case and re-write the argument of the exponential in Eq. (14) (valid in the former case) in the following form:

$F_{\Phi_{0}}=-\frac{2 \mathcal{E} / m-v_{z^{\prime}}^{2}+\left(v_{z^{\prime}}-v_{c \perp} \tan \Phi_{0}\right)^{2}-v_{c \perp}^{2}}{2 v_{\text {th, p }}^{2}}$.

The velocity component $v_{z^{\prime}}$ in this expression can be interpreted as the guiding center velocity component parallel to $\boldsymbol{B}: v_{z^{\prime}}=v_{c \|}$. Therefore, we write

$F_{\Phi_{0}}=-\frac{2 \mathcal{E} / m-v_{c \|}^{2}+\left[v_{c \|}-U_{\|}\left(v_{c \perp}\right)\right]^{2}-v_{c \perp}^{2}}{2 v_{\mathrm{th}, \mathrm{p}}^{2}}$,

where $U_{\|}\left(v_{c \perp}\right)=v_{c \perp} \tan \Phi_{0}$. Expression (28) is valid in any reference frame and, therefore, it is suitable to be generalized as per the nonuniform- $\boldsymbol{B}$ case. Indeed, to obtain such a generalization, we have only to specify another form for the function $U_{\|}\left(v_{c \perp}\right)$, which has the role of generating a bulk velocity component parallel to $\boldsymbol{B}$. In the case of nonuniform $\boldsymbol{B}$, the magnetic field changes direction with $x$ while the bulk velocity $\boldsymbol{u}$ must keep parallel to the $y$ axis for any $x$. Indicating by $\boldsymbol{e}_{\|}=\boldsymbol{B} / \boldsymbol{B}$ and $\boldsymbol{e}_{\perp}=\boldsymbol{e}_{\|} \times \boldsymbol{e}_{x}$ the unit vectors parallel and perpendicular to $\boldsymbol{B}$, we can assume that $\boldsymbol{u} \sim U_{\|} \boldsymbol{e}_{\|}+v_{c \perp} \boldsymbol{e}_{\perp}$. Imposing $u_{z}=0$ in the previous expression we derive the relation $U_{\|}=\left(B_{y} / B_{z}\right) v_{c \perp}=$ $v_{c \perp} \tan \Phi$. On the base of the above considerations, we assume the following form for the DF in the general case:

$$
\begin{aligned}
f_{\Phi(x)}(x, \boldsymbol{v})= & C \exp \left\{-\frac{1}{2 v_{\mathrm{th}, \mathrm{p}}^{2}}\left\{v_{x}^{2}+v_{y}^{2}+v_{z}^{2}+\right.\right. \\
& (2 e / m) \phi\left(x ; x_{c}(x, \boldsymbol{v})\right)-\left[v_{c \|}(x, \boldsymbol{v})\right]^{2}-\left[v_{c \perp}(x, \boldsymbol{v})\right]^{2} \\
& \left.\left.+\left[v_{c \|}(x, \boldsymbol{v})-v_{c \perp}(x, \boldsymbol{v}) \tan \Phi\left(x_{c}(x, \boldsymbol{v})\right)\right]^{2}\right\}\right\} .
\end{aligned}
$$

The associated electron pressure, $p_{\mathrm{e}}$, is calculated by Eq. (4). We show that the bulk velocity component $u_{x}$ is vanishing; moreover, it is $\boldsymbol{j} \times \boldsymbol{B}=0$. Therefore, the only nonvanishing component of Eq. (4) is reduced to

$$
\begin{array}{r}
\frac{\mathrm{d} p_{\mathrm{e}}}{\mathrm{d} x}=-e n(x)\left\{E(x)+\frac{B_{0}}{c}\left[u_{y}(x) \cos \Phi(x)\right.\right. \\
\left.\left.-u_{z}(x) \sin \Phi(x)\right]\right\},
\end{array}
$$

which determines the profile $p_{e}(x)$.

We observe that $f_{\Phi(x)}(x, v)$ is expressed only in terms of the constants of motion: total energy, $\mathcal{E}$, guiding center position, $x_{c}$, and velocity, $\boldsymbol{v}_{c}$. Therefore, the DF (29) is a stationary solution of the Vlasov Eq. (1), provided that the electric and magnetic field remain constant in time. Indeed, using the same arguments as in the case of uniform $\boldsymbol{B}$, it can be shown that $\partial \boldsymbol{E} / \partial t=\partial \boldsymbol{B} / \partial t=$ $\partial p_{e} / \partial t=0$. We conclude that the considered configuration is a stationary solution of the whole set of Eqs. (1)-(4). We also note that expression (29) is reduced to the form (14) when $\Phi(x)=$ $\Phi_{0}=$ const.

For general profiles of $E(x)$ and $\Phi(x)$, an explicit evaluation of the DF (29) can be obtained using the same numerical procedure as in the case when $\Phi(x)=\Phi_{0}=$ const, which determines $f_{\Phi(x)}$ on a $4 \mathrm{D}$ grid in the $\{x, \boldsymbol{v}\}$ phase space. In particular, for the sake of illustration we consider a particular case where $E(x)$ is given by Eq. (19) and

$\Phi(x)=\Delta \Phi\left[\tanh \left(\frac{x-L / 4}{\Delta x}\right)-\tanh \left(\frac{x-3 L / 4}{\Delta x}\right)-1\right]$. 


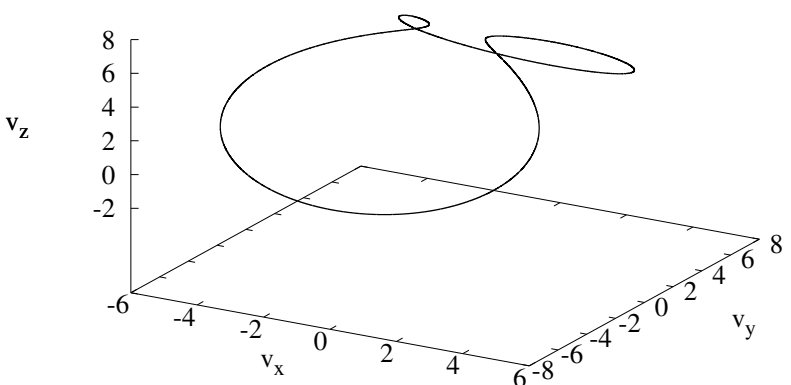

Fig. 8. Trajectory in the velocity space of a particle starting from $x=75$, $v_{x}=-1, v_{y}=0.5, v_{z}=6.5$.

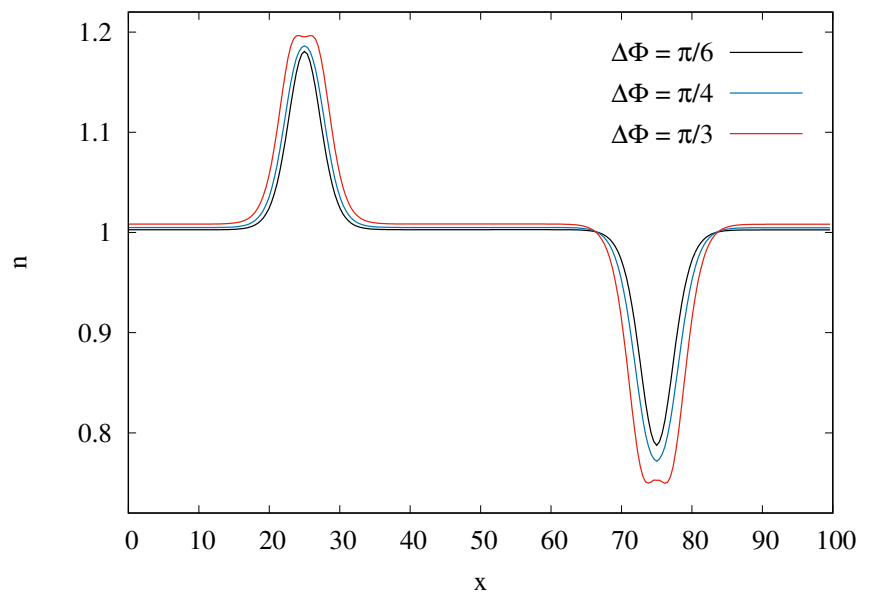

Fig. 9. Profiles of density $n$ as a function of $x$ calculated for $\Delta \Phi=\pi / 6$ (black line), $\Delta \Phi=\pi / 4$ (blue line), and $\Delta \Phi=\pi / 3$ (red line) in the case of nonuniform $\boldsymbol{B}$.

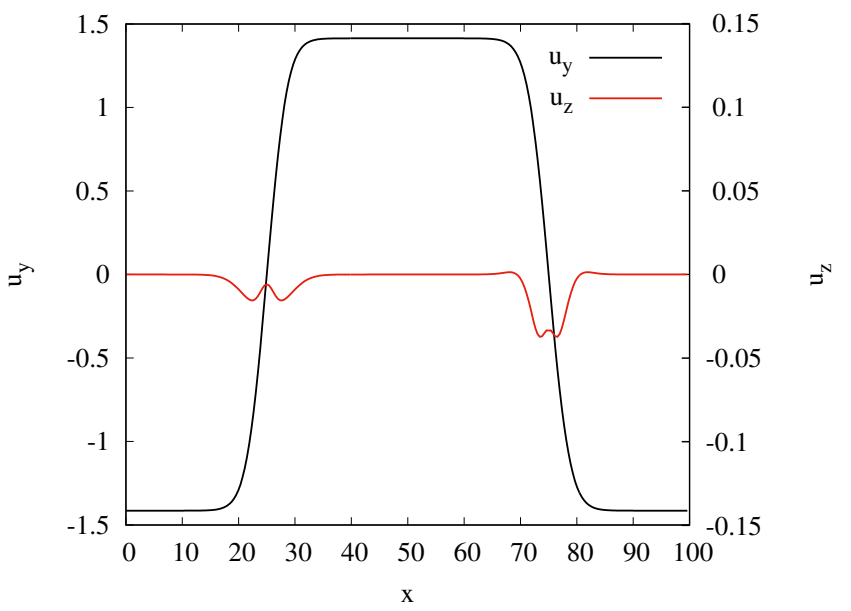

Fig. 10. Profiles of bulk velocity components $u_{y}$ (black line) and $u_{z}$ (red line) as functions of $x$ calculated for $\Delta \Phi=\pi / 4$ in the case of nonuniform $\boldsymbol{B}$. Different scales are used for $u_{y}$ and $u_{z}$.

In this configuration, $\boldsymbol{B}$ rotates by an angle $\pm 2 \Delta \Phi$ across the shear layers, while it is essentially uniform away from the shear layers. The $B_{y}$ component changes sign across the shear layers, while $\boldsymbol{B}$ is in the $z$ direction at the center of each shear layer, $x=L / 4$ and $x=3 L / 4$, where $\Phi=0$. The width $\Delta x$ of the magnetic shears is the same as that of the velocity shears.

Numerical results are presented for $L=100, \Delta x=2.5$ (in normalized units) and for various values of $\Delta \Phi$. In Fig. 8, we plot the trajectory of a particle in the velocity space. The curve is
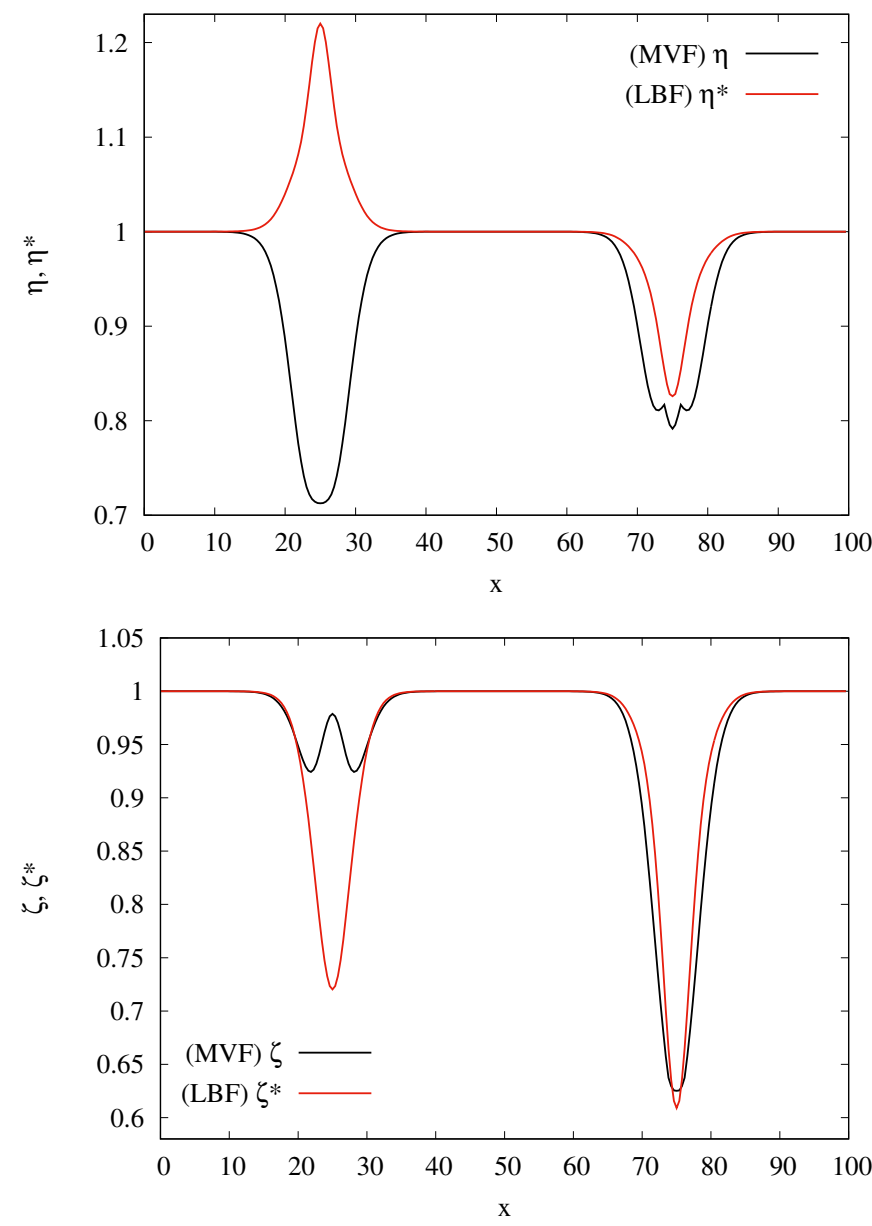

Fig. 11. Upper panel: profiles of anisotropy parameters $\eta$ (black line) and $\eta^{*}$ (red line); lower panel: profiles of agyrotropy parameters $\zeta$ (black line) and $\zeta^{*}$ (red line). Both panels refer to $\Delta \Phi=\pi / 4$ in the nonuniform $\boldsymbol{B}$ case.

more complex than those typically found in the case of uniform $\boldsymbol{B}$ and is not planar. We have calculated the moments of the DF (29) for this configuration. In Fig. 9, the density profile $n(x)$ is plotted; as for the uniform- $\boldsymbol{B}$ case, $n(x)$ has a local maximum or minimum at shear layers, according to the sign of $(\nabla \times \boldsymbol{u})$. $\boldsymbol{\Omega}_{p}$ at shear layers. This asymmetry tends to increase and the profile $n(x)$ becomes more complex for increasing $\Delta \Phi$. For all the values of $\Delta \Phi$, the DF has been normalized by imposing that $\int_{0}^{L} n(x) \mathrm{d} x / L=1$.

In Fig. 10, the profiles of the bulk velocity components $u_{y}(x)$ and $u_{z}(x)$ are plotted, using different scales for the two profiles ( $u_{x}$ is vanishing). The bulk velocity is not exactly aligned along $y$; however, it is $\left|u_{z}(x)\right| \ll\left|u_{y}(x)\right|$. Therefore, the desired condition that $\boldsymbol{u}$ is along the same direction in the whole domain is reasonably well satisfied by the DF (29). In Fig. 11, the anisotropy $\eta, \eta^{*}$ and agyrotropy $\zeta, \zeta^{*}$ parameters are plotted in the case $\Delta \Phi=\pi / 4$. The DF is anisotropic and agyrotropic at the shear layers, with respect to both the MVF and LBF. The profiles of heat flux components $q_{y}$ and $q_{z}$ are plotted in Fig. 12 in the case $\Delta \Phi=\pi / 4$ (it is $q_{x}=0$ ). A peculiar feature is that the $q_{z}$ component averaged across one shear layer $\left\langle q_{z}\right\rangle_{\mathrm{sl}}$ is no longer vanishing; this holds for both shear layers where a net heat flux directed opposite to the mean magnetic field $\langle\boldsymbol{B}\rangle_{\mathrm{sl}}$ has been found. A similar behaviour has been found also for other values of $\Delta \Phi$ (not shown). Finally, in Fig. 13, 3D contour plots of the DF in the 


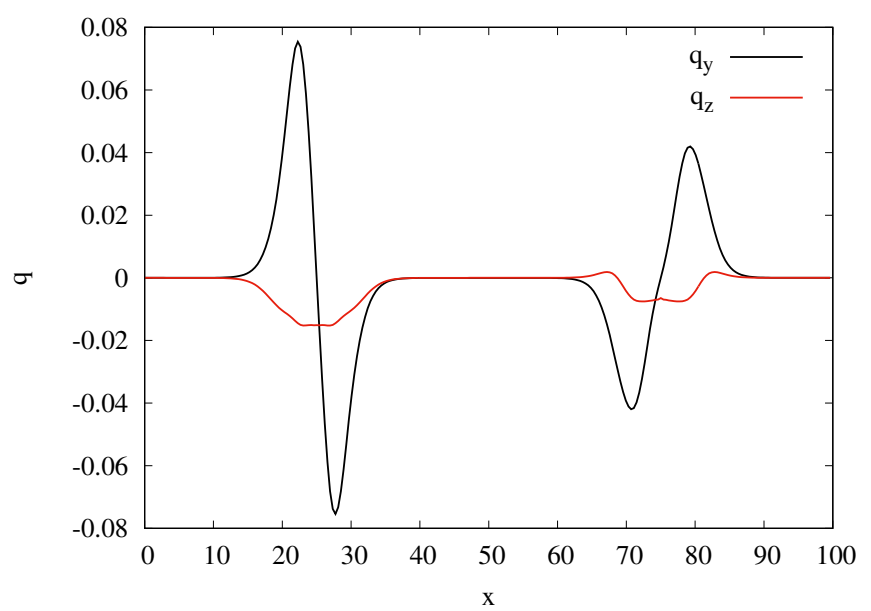

Fig. 12. Profiles of heat flux components $q_{y}$ (black line) and $q_{z}$ (red line) are plotted as functions of $x$, calculated for $\Delta \Phi=\pi / 4$ in the case of nonuniform $\boldsymbol{B}$.

velocity space are plotted at three different positions across one shear layer. Blue arrows indicate the local direction of $\boldsymbol{B}$. Lack of both isotropy and gyrotropy is clearly visible. Moreover, in the position $x=L / 4$ corresponding to the shear layer center (middle panel), an asymmetry with respect to the exchange $v_{z} \rightleftharpoons\left(-v_{z}\right)$ is evident. Such a feature is responsible for the net heat flux in the $-z$ direction.

\section{Conclusions}

In this paper, we derive exact solutions for the system of HVM equations representing a stationary shearing flow in collisionless magnetized plasmas. In many natural contexts, there are configurations where a plasma is characterized by shearing flows. A kinetic description is necessary whenever the shear width is of the order of kinetics scales, as, for instance, in the case of Earth's magnetophause (Sckopke et al. 1981; Kivelson \& Chen 1995; Faganello \& Califano 2017).

The interest of building up stationary solutions can be related to the problem of describing the propagation and evolution of waves in a plasma with a stable shearing flow. The interaction between waves and the background inhomogeneity associated with the shearing flow moves the wave energy toward small scales, where kinetic effects are more effective. Moreover, the presence of a shearing flow can generate wave coupling, with an energy transfer among different wave modes. It is clear that in order to properly study wave propagation, it is necessary for the background structure to remain stationary; otherwise, a time evolution intrinsic of the background state would superpose onto the waves, making it difficult to single out the wave contribution in the overall time evolution. Another possible application of exact shearing flow solutions can be found in the study of KHI, which takes place in unstable shearing velocity configurations. In fact, only a stationary, unperturbed configuration allows us to properly describe both linear and nonlinear stages of the instability, as shown by Settino et al. (2020). Therefore, in both cases, it is crucial to use an exact stationary distribution function.

Stationary solutions in various configurations have been derived in previous studies of the fully kinetic case, that is, involving the full set of ion and electron Vlasov-Maxwell equations (Ganguli et al. 1988; Nishikawa et al. 1988; Cai et al. 1990; Mahajan \& Hazeltine 2000). Allanson et al. (2016) have studied stationary DFs as products of a Maxwellian multi- plied by a combination of Hermite expansions depending on canonical momenta that are constants of motions. Similarly, our most general expression (29) can be factorized as a product of a Maxwellian by a factor that is dependent only on constants of motions. However, our approach presents several differences with respect to that of Allanson et al. (2016). First, our results are relative to the HVM theory, while in the approach by Allanson et al., ions and electrons are both kinetically represented. Moreover, in our case, charge neutrality is only approximately satisfied and an electric field is present that is responsible for plasma drift motion. In contrast, Allanson et al. (2016) consider perfect charge neutrality and null electric field, while the method is applied to calculate equilibria without a bulk velocity. The fully kinetic treatment is quite complex and such solutions have rarely been employed in numerical simulations as, for instance, in investigations of KHI. In this respect, the set of HVM equations represents a good compromise because it correctly describes a plasma at scales of the order of or larger than ion scales but while avoiding the complexity of a fully kinetic treatment.

Within this framework, the exact stationary solutions of Paper I have been set into the special cases where the magnetic field is uniform and either parallel or perpendicular to the bulk velocity. In the present paper, we extend these previous results to more general magnetized shearing flow configurations, namely: (1) uniform obliquely-directed magnetic field; and (2) variabledirected uniform-intensity magnetic field. In the first configuration, the magnetic field, $\boldsymbol{B}$, is uniform and forms a constant angle with the bulk velocity, $\boldsymbol{u}$. In the second configuration the angle between $\boldsymbol{B}$ and $\boldsymbol{u}$ is not constant and changes along the direction perpendicular to $\boldsymbol{u}$ and $\boldsymbol{B}$. Such configurations are more general than those considered in Paper I and they can more realistically represent the magnetic configuration across the magnetopause (e.g., Faganello \& Califano 2017). Therefore, they can be employed as a background state in future studies of KHI in the magnetopause in various magnetic configurations, as we are planning to do.

Stationary DFs have been built up as appropriate combinations of single-particle constants of motions, that have been derived through successive generalizations of those found in Paper I. Stationarity with respect to the whole set of HVM equations has been verified. Explicit analytical forms for the DFs has been derived within the local approximation where both $E(x)$ and $B(x)$ have linear profiles. For more general profiles, a numerical procedure has been set up to integrate single-particle motion, to calculate the corresponding values of involved constants of motions, and to derive the DF on a grid in the phase space. We characterize our results by calculating profiles of moments such as density, bulk velocity, and heat flux. Solving the extended two-fluid model equations, Cerri et al. (2013) found that, starting from an MHD equilibrium with uniform density, the density profile tends to a shape qualitatively similar to that shown in Figs. 2 and 9; namely, a maximum and a minimum located at the shear layer where $(\nabla \times \boldsymbol{u}) \cdot \mathbf{\Omega}_{p}$ is negative or positive, respectively. However, the amplitude of density variation is $\sim 3-4 \%$ in Cerri et al. (2013), while it is $\sim 10-15 \%$ in our case. This discrepancy could be partially due to a slightly different shear width $(\Delta x=3$ in Cerri et al. 2013, $\Delta x=2.5$ in our case) and to the fact that the extended two-fluid model is not exactly equivalent to the HVM model.

We also calculated quantities quantifying departures of the DF from Maxwellianity. In particular, we found that in the shear layers, the DF is characterized by a marked anisotropy and agyrotropy. A peculiar property found in the shear regions is a net 

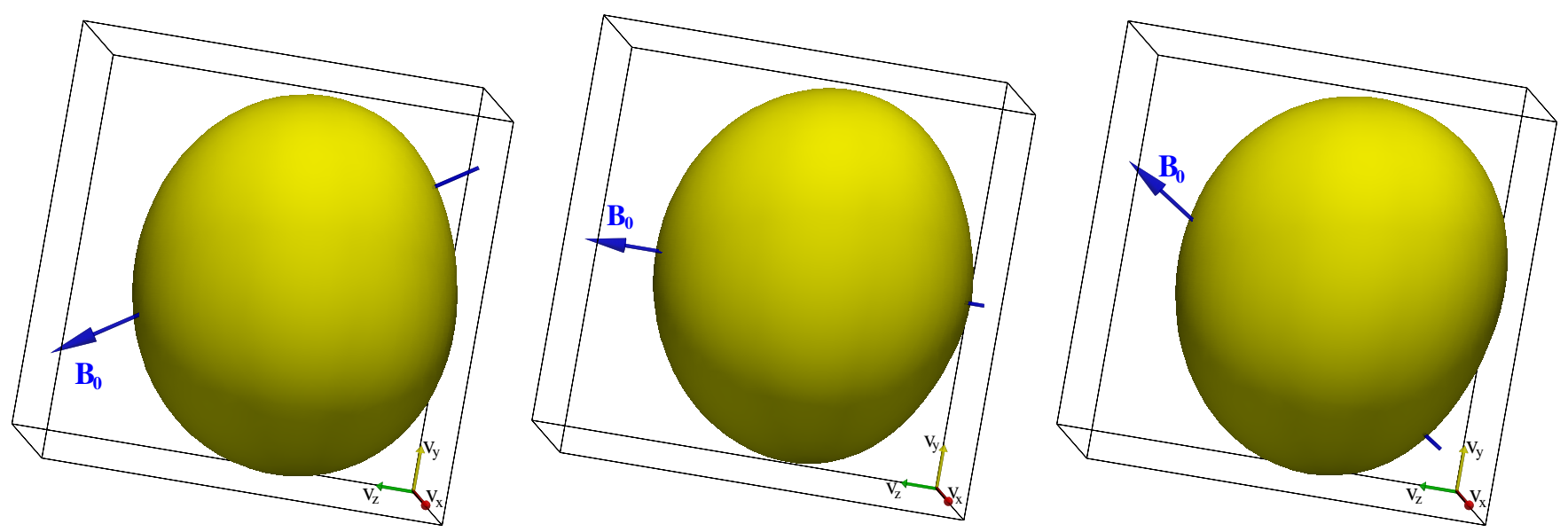

Fig. 13. Iso-surface plots of proton DFs in three different positions along the $x$-axis: $x=L / 4-2.34$ (left panel), $x=L / 4$ (middle panel), $x=L / 4+2.34$ (right panel). The blue arrow indicates the direction of the background magnetic field $\left(B_{0}\right)$.

heat flux in the direction antiparallel to the mean $\boldsymbol{B}$ due to an asymmetry of the DF. We also verified that the obtained bulk velocity presents the desired orientation and planarity to a very good extent in both cases. A numerical simulation performed by means of the HVM code has allowed us to verify that departures from stationarity due to numerical errors remain at a low level, at least in the uniform $\boldsymbol{B}$ case. In particular, the width $\Delta x$ of both shear layers remains essentially unchanged. This aspect is particularly relevant in the perspective of using the present stationary solutions to study the KHI. In fact, the growth rate as well as the wavelength of the fastest growing mode are affected by the value of the shear layer width, $\Delta x$. For instance, Nakamura et al. (2010) and Henri et al. (2013) showed that initializing a kinetic code with a shifted Maxwellian not only produces spurious oscillations but also a widening or thinning of the shear layer, depending on the relative orientation of $\nabla \times \boldsymbol{u}$ and $\boldsymbol{B}$. Our solution should allow future studies to overcome such a problem.

Further possible developments of the present work that we are planning to carry out involve the inclusion of inhomogeneities of magnetic field intensity $|\boldsymbol{B}|$. This would allow us to perform a further step towards a more realistic representation of configurations, such as those found around the magnetopause. We also plan to use the configurations considered here to simulate the development of KHI in situations where kinetic effects at ion scales can play an important role.

Acknowledgements. Numerical simulations have been run on Marconi supercomputer at CINECA (Italy) within the ISCRA projects: IsC68_TURB-KHI and IsB19_6DVLAIDA. This work has received funding from the European Unions Horizon 2020 research and innovation programme under grant agreement no. 776262 (AIDA, www .aida-space.eu).

\section{References}

Allanson, O., Neukirch, T., Troscheit, S., \& Wilson, F. 2016, J. Plasma Phys., 82, 905820306

Axford, W. I. 1960, QJMAM, 8, 314

Bruno, R., \& Carbone, V. 2013, Liv. Rev. Sol. Phys., 10, 2

Cai, D., Storey, L. R. O., \& Neubert, T. 1990, PhFlB, 2, 75

Califano, F., Chiuderi, C., \& Einaudi, G. 1990, ApJ, 365, 757

Califano, F., Chiuderi, C., \& Einaudi, G. 1992, ApJ, 390, 560

Cerri, S. S., Henri, P., Califano, F., et al. 2013, Phys. Plasmas, 20, 112112

Cerri, S. S., Pegoraro, F., Califano, F., Del Sarto, D., \& Jenko, F. 2014, PhPl, 21, 112109
Cerri, S. S., Califano, F., Jenko, F., Told, D., \& Rincon, F. 2016, ApJ, 822, L12 Cerri, S. S., Servidio, S., \& Califano, F. 2017, ApJ, 846, L18

Chandrasekhar, S. 1961, Hydrodynamic and Hydromagnetic Stability (Oxford University Press)

Contin, J. E., Gratton, F. T., \& Farrugia, C. J. 2003, JGRA, 108, 1227

Cowee, M. M., \& Winske, D.,\& Gary, S. P., 2009, JGRA, 4, A10209

Del Sarto, D., Pegoraro, F., \& Califano, F. 2016, PhRvE, 93, 053203

Eriksson, S., Lavraud, B., Wilder, F. D., et al. 2016, GeoRL, 43, 5606

Ershkovich, A. I., \& Mendis, D. A. 1983, ApJ, 269, 743

Faganello, M., \& Califano, F. 2017, JPIPh, 83, 535830601

Faganello, M., Califano, F., \& Pegoraro, F. 2008, PhRvL, 100, 015001

Fairfield, D. H., Otto, A., Mukai, T., et al. 2000, JGRA, 105, 21159

Fairfield, D. H., Farrugia, C. J., Mukai, T., Nagai, T., \& Fedorov, A. 2003, JGRA, 108,1460

Foullon, C., Verwichtel, E., Nakariakov, V. M., Nykyri, K., \& Farrugia, C. J. 2011, ApJ, 729, L8

Franci, L., Verdini, A., Matteini, L., Landi, S., \& Hellinger, P. 2015, ApJ, 804, L39

Fujimoto, M., Terasawa, T., Mukai, T., et al. 1998, J. Geophys. Res., 103, 4391 Ganguli, G., Lee, Y. C., \& Palmadesso, P. J. 1988, PhFl, 31, 823

Hamlin, N. D., \& Newman, W. I. 2013, Phys. Rev. E, 87, 043101

Hasegawa, H., Fujimoto, M., Phan, T.-D., et al. 2004, Nature, 430, 755

Hasegawa, H., Fujimoto, M., Takagi, K., et al. 2006, JGRA, 111, 9203

Henri, P., Cerri, S. S., Califano, F., et al. 2013, PhPl, 20, 102118

Hollweg, J. V. 1987, ApJ, 312, 880

Kaghashvili, E. K. 1999, ApJ, 512, 969

Kaghashvili, E. K., Raeder, J., Webb, G. M., \& Zank, G. P. 2006, Phys. Plasmas, 13, 112107

Kaghashvili, E. K. 2007, Phys. Plasmas, 14, 044502

Karimabadi, H., Roytershteyn, V., Wan, M., et al. 2013, JPIPh, 20, 012303

Kivelson, M. G., \& Chen, S. 1995, in Physics of the Magnetopause (Washington, DC: AGU), Geophys. Monogr. Ser., 90

Landi, S., Velli, M., \& Einaudi, G. 2005, ApJ, 624, 392

Lee, M., \& Roberts, B. 1986, ApJ, 301, 430

Mahajan, S. M., \& Hazeltine, R. D. 2000, PhPl, 7, 1287

Maiorano, T., Settino, A., Malara, F., et al. 2020, J. Plasma Phys., 86, 825860202

Malara, F., Einaudi, G., \& Mangeney, A. 1989, J. Geophys. Res., 94, 11805

Malara, F., Veltri, P., Chiuderi, C., \& Einaudi, G. 1992, ApJ, 396, 297

Malara, F., Primavera, L., \& Veltri, P. 1996a, ApJ, 459, 347

Malara, F., Primavera, L., \& Veltri, P. 1996b, J. Geophys. Res., 101, 21597

Malara, F., Petkaki, P., \& Veltri, P. 2000, ApJ, 533, 523

Malara, F., De Franceschis, M. F., \& Veltri, P. 2003, A\&A, 412, 529

Malara, F., De Franceschis, M. F., \& Veltri, P. 2005, A\&A, 443, 1033

Malara, F., De Franceschis, M. F., \& Veltri, P. 2007, A\&A, 467, 1275

Malara, F., Pezzi, O., \& Valentini, F. 2018, Phys. Rev. E, 97, 053212

Marsch, E. 2006, Liv. Rev. Solar Phys., 3, 1

Matsumoto, Y., \& Hoshino, M. 2004, GeoRL, 31, L02807

Matsumoto, Y., \& Hoshino, M. 2006, JGRA, 111, A05213

Matsumoto, Y., \& Seki, K. 2010, JGRA, 115, A10231

Matthaeus, W. H., Oughton, S., Osman, K. T., et al. 2014, APJ, 790, 155

McComas, D. J., Gosling, J. T., Bame, S. J., et al. 1987, J. Geophys. Res., 92, 1139

Miura, A. 1982, PhRvL, 19, 779 
Miura, A. 1990, Geophys. Res. Lett., 17, 749

Mok, Y., \& Einaudi, G. 1985, J. Plasma Phys., 33, 199

Nakamura, T. K. M., Hayashi, D., Fujimoto, M., \& Shinohara, I. 2004, PhRvL, 92,145001

Nakamura, T. K. M., Hasegawa, H., \& Shinohara, I. 2010, PhPl, 17, 042119

Nakamura, T. K. M., Hasegawa, H., Shinohara, I., \& Fujimoto, M. 2011, JGRA, 116, A03227

Nakamura, T. K. M., Daughton, W., Karimabadi, H., \& Eriksson, S. 2013, JGRA 118,5742

Nakamura, T. K. M., Hasegawa, H., Daughton, W., et al. 2017, NatCo, 8, 1582

Nishikawa, K.-I., Ganguli, G., Lee, Y. C., \& Palmadesso, P. J. 1988, PhFl, 31, 1568

Nykyri, K., Otto, A., Lavraud, B., et al. 2006, AnGeo, 24, 2619

Petkaki, P., Malara, F., \& Veltri, P. 1998, ApJ, 500, 483

Pezzi, O., Parashar, T. N., Servidio, S., et al. 2017a, J. Plasma Phys., 83, 1

Pezzi, O., Parashar, T. N., Servidio, S., et al. 2017b, ApJ, 834, 166

Pezzi, O., Servidio, S., Perrone, D., et al. 2018, Phys. Plasmas, 25, 060704

Pritchett, P. L., \& Coroniti, F. V. 1984, JGR, 89, 168

Pucci, F., Onofri, M., \& Malara, F. 2014, ApJ, 796, 43
Pucci, F., Vàsconez, C. L., Pezzi, O., et al. 2016, J. Geophys. Res., 121, 1024 Roberts, D. A., Goldstein, M. L., Matthaeus, W. H., \& Ghosh, S. 1992, J. Geophys. Res., 97, 17115

Roytershteyn, V., \& Daughton, W. 2008, PhPl, 15, 082901

Sckopke, N., Paschmann, G., Haerendel, G., et al. 1981, J. Geophys. Res., 86, 2099

Seon, J., Frank, L. A., Lazarus, A. J., \& Lepping, R. P. 1995, J. Geophys. Res., 100, 11907

Servidio, S., Valentini, F., Califano, F., \& Veltri, P. 2012, PhRvL, 108, 045001

Servidio, S., Valentini, F., Perrone, D., et al. 2015, JPIPh, 81, 325810107

Settino, A., Malara, F., Pezzi, O., et al. 2020, ApJ, 901, 17

Sisti, M., Faganello, M., Califano, F., et al. 2019, GeoRL, 46(11), 597

Tsiklauri, D., Nakariakov, V., \& Rowlands, G. 2002, A\&A, 393, 321

Valentini, F., Trávníček, F., Califano, P., et al. 2007, ApJ, 770, 225

Valentini, F., Perrone, D., \& Veltri, P. 2011, ApJ, 739, 54

Valentini, F., Perrone, D., Stabile, S., et al. 2016, NJPh, 18, 125001

Valentini, F., Vàsconez, C. L., Pezzi, O., et al. 2017, A\&A, 599, A8

Vàsconez, C. L., Pucci, F., Valentini, F., et al. 2015, ApJ, 815, 7

Walker, A. D. M. 1981, P\&SS, 29, 1119 


\section{Appendix A: Local approximation for uniform oblique $B$}

In this appendix, we focus a more detailed study on a configuration where the electric field has the linear profile of (15), which can be considered as a local approximation for a general profile $E(x)$ around the position $x_{0}$. In particular, we derive the expressions for the guiding center velocity and for the DF (Eq. (16)). The form for the magnetic field is given by Eq. (5) with $\Phi(x)=\Phi_{0}=$ const. With respect to the reference frame $S^{\prime}$, the normalized vector potential has the form $\boldsymbol{a}(x)=x \boldsymbol{e}_{y^{\prime}}$. In this case, Eq. (8) becomes $d^{2} x / \mathrm{d} t^{2}+\omega^{2} x=(e / m)\left(E_{0}-\alpha_{0} x_{0}\right)+\Omega_{p} w_{y^{\prime}}$ where $\omega^{2}=\Omega_{p}^{2}-e \alpha_{0} / m$ and $w_{y^{\prime}}=v_{y^{\prime}}+\Omega_{p} x$ is a constant of motion. If $\alpha_{0}<m \Omega_{p}^{2} / m$, the above equation describes an harmonic oscillator of solution

$x(t)=R_{0} \sin (\omega t+\varphi)+x_{c}$,

where $R_{0}$ and $\varphi$ are the amplitude and the phase of the oscillation.

The guiding center position is given by the constant term:

$x_{c}=\langle x\rangle_{\tau}=\frac{1}{\omega^{2}}\left[\frac{e}{m}\left(E_{0}-\alpha_{0} x_{0}\right)+\Omega_{p} w_{y^{\prime}}\right]$,

that can be expressed as a function of $x$ and $v_{y^{\prime}}$ :

$x_{c}=\frac{1}{1-c \alpha_{0} /\left(\Omega_{p} B_{0}\right)}\left[x-\frac{v_{y^{\prime}}}{\Omega_{p}}+\frac{c}{\Omega_{p} B_{0}}\left(E_{0}-\alpha_{0} x_{0}\right)\right]$.

The velocity components are given by

$v_{x}(t)=R_{0} \omega \cos (\omega t+\varphi)$,

$v_{y^{\prime}}(t)=-\Omega_{p} x(t)+w_{y^{\prime}}=-R_{0} \Omega_{p} \sin (\omega t+\varphi)+v_{c \perp}$,

and $v_{z^{\prime}}(t)=$ const, where $v_{c \perp}=\left\langle v_{y^{\prime}}(t)\right\rangle_{\tau}=-\Omega_{p} x_{c}+w_{y^{\prime}}$. By inserting Eq. (A.2) into this expression, we obtain $v_{c \perp}=$ $-\left[e /\left(m \omega^{2}\right)\right]\left(\Omega_{p} E_{0}+\alpha_{0} v_{0 y^{\prime}}\right)$, where $v_{0 y^{\prime}}=-\Omega_{p} x_{0}+w_{y^{\prime}}$ is the value of $v_{y^{\prime}}$ at the position $x_{0}$. On the other hand, with $w_{y^{\prime}}$ as a constant of motion, it is $v_{0 y^{\prime}}=v_{c \perp}+\Omega_{p}\left(x_{c}-x_{0}\right)$ (see Eq. (7)). Using this relation, from the above equations, we derive:

$v_{c \perp}=-\frac{e}{m \omega}\left[E_{0}+\alpha_{0}\left(x_{c}-x_{0}\right)\right]=-c \frac{E\left(x_{c}\right)}{B_{0}}$,

indicating that the guiding center velocity component perpendicular to $\boldsymbol{B}$ is given by the $\boldsymbol{E} \times \boldsymbol{B}$ drift velocity calculated at the guiding center position $x_{c}$.

The argument of the exponential in Eq. (14) contains the constant of motion $2 \mathcal{E}_{\perp} / m-v_{c \perp}^{2}=v_{x}^{2}+v_{y^{\prime}}^{2}+(2 e / m) \phi-v_{c \perp}^{2}$. Such a constant can be evaluated at $x=x_{c}$, where the potential is $\phi=0$; at that position, it is $\sin (\omega t+\varphi)=0$ and then $v_{x}\left(x=x_{c}\right)= \pm R_{0} \omega$ and $v_{y^{\prime}}\left(x=x_{c}\right)=v_{c \perp}$ (see Eqs. (A.4) and (A.5)). As a consequence, it is $2 \mathcal{E}_{\perp} / m-v_{c \perp}^{2}=R_{0}^{2} \omega^{2}$ and, thus, Eq. (14) can be written as:

$f_{\Phi_{0}}^{l a}=C \exp \left[-\frac{R_{0}^{2} \omega^{2}+\left(v_{z^{\prime}}-v_{c \perp} \tan \Phi_{0}\right)^{2}}{2 v_{\mathrm{th}, \mathrm{p}}^{2}}\right]$.

Using Eqs. (A.4), (A.5), and (A.6), the constant $R_{0}^{2} \omega^{2}$ can be expressed as a function of $x, v_{x}$ and $v_{y^{\prime}}$ in the following form:

$R_{0}^{2} \omega^{2}=v_{x}^{2}+\frac{\omega^{2}}{\left(\Omega_{p}-c \alpha_{0} / B_{0}\right)^{2}}\left(v_{y^{\prime}}+\frac{c E(x)}{B_{0}}\right)^{2}$.
From Eqs. (A.6) and (A.3), we can express $v_{c \perp}$ as a function of $x$ and $v_{y^{\prime}}$ :

$v_{c \perp}=v_{d 0}-\frac{c \alpha_{0}}{\Omega_{p} B_{0}-c \alpha_{0}}\left[\Omega_{p}\left(x-x_{0}\right)+v_{y}-v_{d 0}\right]$,

where $v_{d 0}=-c E_{0} / B_{0}$ is the drift velocity at the position $x_{0}$. By inserting Eqs. (A.8) and (A.9) into Eq. (A.7), this can be manipulated and, finally, written in the form of (16), where the normalization constant $C$ has been determined by imposing $\int f_{\Phi_{0}}^{l a} d^{3} v=n_{0}$. A straightforward calculation allows us to find Eq. (17).

\section{Appendix B: Local approximation for uniform-intensity variable-direction $B$}

In this appendix, we derive the form for the DF in the case of the local approximation for a magnetic field with uniform intensity and variable direction. The electric field takes the form of Eq. (15) and the magnetic field components have linear profiles given by Eqs. (23) and (24), where $x_{0}$ represents the center of the Taylor expansion. The corresponding potentials are

$\phi(x)=-E_{0}\left(x-x_{0}\right)-\frac{\alpha_{0}}{2}\left(x-x_{0}\right)^{2}+\phi_{0}$,

$a_{y}(x)=b_{0 z}\left(x-x_{0}\right)-\frac{\gamma_{z}}{2}\left(x-x_{0}\right)^{2}, a_{z}(x)=-b_{0 y}\left(x-x_{0}\right)-\frac{\gamma_{y}}{2}\left(x-x_{0}\right)^{2}$,

with $\phi_{0}=$ const. Imposing the $|\boldsymbol{B}(x)|=B_{0}=$ uniform, at the linear order we obtain the relations:

$b_{0 y}^{2}+b_{0 z}^{2}=1, \quad b_{0 y} \gamma_{y}=b_{0 z} \gamma_{z}$.

In coherence with the local approximation, in what follows, we neglect all terms cubic or of higher order in the ratios $\left|\alpha_{0}\left(x-x_{0}\right) / E_{0}\right|$ and $\left|\gamma_{y, z}\left(x-x_{0}\right) / b_{0 y, 0 z}\right|$. The single particle equation of motion in the $x$ direction takes the form $d^{2} x / \mathrm{d} t^{2}+\omega^{2} x=$ $(e / m)\left(E_{0}-\alpha_{0} x_{0}\right)+\Omega_{p} W_{0}$, where $\omega$ is given by Eq. (25) and

$W_{0}=\left(w_{y} \gamma_{z}+w_{z} \gamma_{y}\right) x_{0}+w_{y} b_{0 z}-w_{z} b_{0 y}+\Omega_{p} x_{0}$.

If $\alpha_{0}<(m / e)\left[\Omega_{p}^{2}+\Omega_{p}\left(w_{y} \gamma_{z}+w_{z} \gamma_{y}\right)\right]$ it is $\omega^{2}>0$ and the particle motion along $x$ is that of an harmonic oscillator,

$x(t)=R_{0} \sin (\omega t+\varphi)+x_{c}, \quad v_{x}(t)=R_{0} \omega \cos (\omega t+\varphi)$,

where $R_{0}$ is the amplitude, $\varphi$ is the phase and $x_{c}$ is the center of motion,

$$
\begin{aligned}
x_{c}= & \frac{1}{\omega^{2}}\left\{\frac{e}{m}\left(E_{0}+\alpha_{0} x_{0}\right)+\right. \\
& \left.+\Omega_{p}\left[w_{y}\left(b_{0 z}+\gamma_{z} x_{0}\right)-w_{z}\left(b_{0 y}-\gamma_{y} x_{0}\right)+\Omega x_{0}\right]\right\},
\end{aligned}
$$

corresponding to the $x$ position of the guiding center. Otherwise, if $\alpha_{0} \geq(m / e)\left[\Omega_{p}^{2}+\Omega_{p}\left(w_{y} \gamma_{z}+w_{z} \gamma_{y}\right)\right]$ the motion along $x$ is not bounded and the local approximation breaks down. On the other hand, the motion equation along $x$ can be re-written in the form $\mathrm{d} v_{x}(t) / \mathrm{d} t=(e / m) E(x(t))+$ $\Omega_{p}\left\{v_{y}\left[\mathrm{~d} a_{y}(x(t)) / \mathrm{d} x\right]+v_{z}\left[d a_{z}(x(t)) / d x\right]\right\}$. By inserting Eq. (B.5) into both sides and neglecting third-order terms, we obtain the following relation: 
$-c E\left(x_{c}\right)=w_{y} B_{z}\left(x_{c}\right)-w_{z} B_{y}\left(x_{c}\right)-\Omega_{p} B_{0}\left(x_{c}-x_{0}\right)$.

(B.7)

Using Eqs. (7), (B.2), and (B.5), we derive expressions for $v_{y}$ and $v_{z}$ :

$v_{y}(t)=w_{y}-\Omega_{p} a_{y}\left(x_{c}\right)-\frac{\Omega_{p} B_{z}\left(x_{c}\right)}{B_{0}} R_{0} \sin (\omega t+\varphi)+\frac{\Omega_{p} \gamma_{z}}{2} R_{0}^{2} \sin ^{2}(\omega t+\varphi)$,

$v_{z}(t)=w_{z}-\Omega_{p} a_{z}\left(x_{c}\right)+\frac{\Omega_{p} B_{y}\left(x_{c}\right)}{B_{0}} R_{0} \sin (\omega t+\varphi)+\frac{\Omega_{p} \gamma_{y}}{2} R_{0}^{2} \sin ^{2}(\omega t+\varphi)$.

We observe that, as a consequence of the dependence on $\sin ^{2}(\omega t+\varphi)$ in $v_{y}(t)$ and $v_{z}(t)$, particle trajectories in the velocity space are no longer ellipses but have a more complex form. The $y$ and $z$ components of the guiding center velocity are

$$
v_{c y}=\left\langle v_{y}\right\rangle_{\tau}=\frac{\Omega_{p} \gamma_{z}}{2}\left(x_{c}-x_{0}\right)^{2}-\Omega_{p} b_{0 z}\left(x_{c}-x_{0}\right)+\frac{\Omega_{p} R_{0}^{2} \gamma_{z}}{4}+w_{y},
$$

$v_{c z}=\left\langle v_{z}\right\rangle_{\tau}=\frac{\Omega_{p} \gamma_{y}}{2}\left(x_{c}-x_{0}\right)^{2}+\Omega_{p} b_{0 y}\left(x_{c}-x_{0}\right)+\frac{\Omega_{p} R_{0}^{2} \gamma_{y}}{4}+w_{z}$.

From these equations we derive expressions for $w_{y}$ and $w_{z}$ that are inserted into Eq. (B.7). Neglecting third-order terms, we obtain $v_{c z} B_{y}\left(x_{c}\right)-v_{c y} B_{z}\left(x_{c}\right)=c E\left(x_{c}\right)$, which determines the component of the guiding center velocity perpendicular to $\boldsymbol{B}\left(x_{c}\right)$ :

$\boldsymbol{v}_{c \perp}=c \frac{\boldsymbol{E}\left(x_{c}\right) \times \boldsymbol{B}\left(x_{c}\right)}{B^{2}}$,

which corresponds to the $\boldsymbol{E} \times \boldsymbol{B}$ drift velocity calculated at the guiding center position $x_{c}$; this is Eq. (26). 